\title{
A REPRESENTATION THEOREM FOR CYCLIC ANALYTIC TWO-ISOMETRIES
}

\author{
STEFAN RICHTER
}

\begin{abstract}
A bounded linear operator $T$ on a complex separable Hilbert space $\mathscr{H}$ is called a 2-isometry if $T^{* 2} T^{2}-2 T^{*} T+I=0$. We say that $T$ is analytic if $\bigcap_{n>0} T^{n} \mathscr{H}=(0)$. In this paper we show that every cyclic analytic 2 -isometry can be represented as multiplication by $z$ on a Dirichlet-type space $D(\mu)$. Here $\mu$ denotes a finite positive Borel measure on the unit circle. For two measures $\mu$ and $\nu$ the 2-isometries obtained as multiplication by $z$ on $D(\mu)$ and $D(\nu)$ are unitarily equivalent if and only if $\mu=\nu$. We also investigate similarity and quasisimilarity of these 2 -isometries, and we apply our results to the invariant subspaces of the Dirichlet shift.
\end{abstract}

\section{INTRODUCTION}

Let $T$ be a bounded linear operator on a complex separable Hilbert space $\mathscr{H}$. Following J. Agler [1] we say that $T$ is a 2-isometry if

$$
T^{* 2} T^{2}-2 T^{*} T+I=0 .
$$

Using quadratic forms we see that $T$ is a 2-isometry if and only if

$$
\left\|T^{2} x\right\|^{2}-2\|T x\|^{2}+\|x\|^{2}=0 \text { for every } x \in \mathscr{X} .
$$

It follows that every isometry is a 2-isometry. The Dirichlet shift is an example of an operator that is a 2-isometry, but not an isometry.

A study of 2-isometries and related operators is of interest for several reasons. First, even though the spectrum of any 2 -isometry must be contained in the closed unit disc, the operator must always expand the norm of every vector in the space. Thus, the 2-isometries are examples of operators which are not members of classes which have been extensively studied like, e.g., the class of contractions or subnormal operators. Secondly, as 2-isometries are generalizations of isometries, we shall show in a forthcoming paper that they can be used as dilations for another class of expanding operators.

Our current interest in 2-isometries is based on the observation that, if $T$ is a 2-isometry and $\mathscr{M}$ is a nonzero invariant subspace of $T$, then $T \mid \mathscr{M}$ is a 2-isometry as well. This fact was one of the key ideas that enabled the author in [9] to show that every invariant subspace of the Dirichlet shift is cyclic.

Received by the editors January 19, 1989, and in revised form, September 18, 1989.

1980 Mathematics Subject Classification (1985 Revision). Primary 47B37, 47B38; Secondary $31 \mathrm{C} 25,46 \mathrm{E} 20,47 \mathrm{~A} 67$. 
We also mention that recent work of Agler [1] provides a different kind of motivation for the study of 2-isometries.

We call an operator $T \in \mathscr{L}(\mathscr{H})$ analytic if $\bigcap_{n>0} T^{n} \mathscr{H}=\{0\}$. The intuitive idea behind this definition is that, if $\mathscr{H}$ is a Hilbert space of analytic functions on a domain containing the origin and if $M_{z}$ denotes the operator of multiplication by $z$ acting on $\mathscr{H}$, then $M_{z}$ is analytic. The study of analytic 2-isometries is a natural starting point for a more general study of 2-isometries, because, as we shall show in forthcoming work, every 2-isometry can be uniquely written as the direct sum of a unitary operator and an analytic 2-isometry.

The main result of this paper (Theorem 5.1) is that every cyclic analytic 2isometry can be represented as multiplication by $z$ on a Dirichlet-type space $D(\mu)$. The space $D(\mu)$ depends on a positive Borel measure $\mu$ supported on the unit circle $\mathbb{T}$. This dependence is such that two multiplication operators $M_{z}$ acting on two spaces $D(\mu)$ and $D(\nu)$ are unitarily equivalent if and only if $\mu=\nu$.

Using this representation theorem we shall investigate similarity and quasisimilarity of cyclic analytic 2 -isometries $(\S 6)$. In $\S 7$ we shall apply our results to invariant subspaces of the Dirichlet shift. For example, we shall show that every invariant subspace of the Dirichlet shift is of the form $f D\left(m_{f}\right)$, where $f$ is a Dirichlet space function and $m_{f}$ is an absolutely continuous measure associated with $f$.

In [6] Halmos asked the following question: If $T$ is a weighted shift operator and if $\mathscr{M}$ is a nonzero invariant subspace of $T$, is $T \mid \mathscr{M}$ similar to a weighted shift operator? This question was motivated by the observation that the answer is yes for the unilateral shift. Furthermore, if $T$ can be represented as multiplication by $z$ on a Hilbert space of analytic functions on $\mathbb{D}$, if $T-\lambda$ is bounded below for every $\lambda \in \mathbb{D}$, and if $\mathscr{M}$ is generated by a finite Blaschke product, then $T \mid \mathscr{M}$ is similar to a weighted shift operator. It has been known for quite some time that the answer to the question in general is no. However, it remained open in the special case when $T$ is the Dirichlet shift (see [11, p. 109]). In Theorem 7.3 we shall see that the restriction of the Dirichlet shift to an invariant subspace $\mathscr{M}$ is similar to a weighted shift operator if and only if $\mathscr{M}$ is generated by a finite Blaschke product.

\section{Preliminaries}

All Hilbert spaces referred to in this paper are separable. Although we use $m$ to denote Lebesgue measure on $\mathbb{T}$, we shall write $d t$ instead of $d m(t)$.

On various occasions throughout the paper we shall refer to the $D_{\alpha}$-spaces, $\alpha \in \mathbb{R}$, of analytic functions on the open unit disc. The norm on $D_{\alpha}$ can be given in terms of the Taylor coefficients: if $f(z)=\sum \hat{f}(n) z^{n}$, then $\|f\|_{\alpha}^{2}=$ $\sum(n+1)^{\alpha}|\hat{f}(n)|^{2}$. For $\alpha=0$ one has $D_{0}=H^{2}$ and for $\alpha=1 D_{1}=D$, the Dirichlet space. It is easy to verify that $f \in D_{\alpha}$ if and only if $f^{\prime} \in D_{\alpha-2}$. For 
$\alpha<0$ an equivalent norm is given by

$$
\iint_{\mathbb{D}}|f(z)|^{2}\left(1-|z|^{2}\right)^{-1-\alpha} d A(z) .
$$

For the details see [13, Lemma 2]. In particular, we note that the $H^{2}$-norm of a function $f$ is equivalent to $|f(0)|^{2}+\iint_{\mathbb{D}}\left|f^{\prime}(z)\right|^{2}\left(1-|z|^{2}\right) d A(z)$.

We shall now review a few properties of 2-isometries, which are essentially contained in [9, §2]. Lemma 1 of [9] implies that for every 2-isometry $T$ one has $T^{*} T-I \geq 0$. Thus we can define $D$ to be the positive square root of $T^{*} T-I$. The following lemma follows from formula (11) of [9].

Lemma 2.1 [9]. If $T \in \mathscr{L}(\mathscr{H})$ is an analytic 2-isometry and $x_{0} \in \operatorname{ker} T^{*}$, $\left\|x_{0}\right\|=1$, then

$$
\begin{aligned}
\left\|p(T) x_{0}\right\|^{2} & =\|p\|_{H^{2}}^{2}+\sum_{k=1}\left\|D p_{k}(T) x_{0}\right\|^{2} \\
& =\|p\|_{H^{2}}^{2}+\sum_{k=1}\left(\left\|T p_{k}(T) x_{0}\right\|^{2}-\left\|p_{k}(T) x_{0}\right\|^{2}\right),
\end{aligned}
$$

for every polynomial $p$. Here for $k \geq 0$ we used the notation

$$
p_{k}(z)=\sum_{n=k} \hat{p}(n) z^{n} .
$$

Proof. It is clear from the definition of $D$ that the two right-hand sides of $(2.1)$ are equal to one another. Thus we only have to verify the first equality.

Let $L$ be the left inverse of $T$ with $\operatorname{ker} L=\operatorname{ker} T^{*}$ and let $Q$ be the projection onto $\operatorname{ker} T^{*}$. Then for all $k \geq 0$ we have $Q L^{k} p(T) x_{0}=\hat{p}(k) x_{0}$ and $T^{k} L^{k} p(T) x_{0}=p_{k}(T) x_{0}$. The fact that $T$ is a 2-isometry implies that $\left\|D T^{k} x\right\|=\|D x\|$ for every $x \in \mathscr{H}$. Now (2.1) follows from formula (11) of [9] applied with $\mathscr{M}=\mathscr{H}$ and $x=p(T) x_{0}$ :

$$
\begin{aligned}
\left\|p(T) x_{0}\right\|^{2} & =\sum_{k=0}\left\|Q L^{k} p(T) x_{0}\right\|^{2}+\sum_{k=1}\left\|D L^{k} p(T) x_{0}\right\|^{2} \\
& =\sum_{k=0}|\hat{p}(k)|^{2}\left\|x_{0}\right\|^{2}+\sum_{k=1}\left\|D T^{k} L^{k} p(T) x_{0}\right\|^{2} \\
& =\|p\|_{H^{2}}^{2}+\sum_{k=1}\left\|D p_{k}(T) x_{0}\right\|^{2} .
\end{aligned}
$$

\section{THE SPACES $D(\mu)$}

In this section we state and prove the basic facts about $D(\mu)$. We shall see that $D(\mu)$ is always contained in $H^{2}$, that multiplication by $z$ defines a bounded linear operator on $D(\mu)$, that this operator is a 2-isometry, and that the polynomials are dense in $D(\mu)$. 
Let $\mu$ be a positive finite Borel measure on the unit circle $\mathbb{T}=\{z \in \mathbb{C}:|z|=$ $1\}$ and let $P\left(z, e^{i t}\right)=\left(1-|z|^{2}\right) /\left|e^{i t}-z\right|^{2}$ denote the Poisson kernel. Define the positive harmonic function $\varphi_{\mu}$ on the open unit disc $\mathbb{D}$ by

$$
\varphi_{\mu}(z)=\frac{1}{2 \pi} \int_{0}^{2 \pi} P\left(z, e^{i t}\right) d \mu(t) .
$$

If $\mu=0$, then let $D(\mu)=H^{2}$; otherwise we define the space $D(\mu)$ to consist of all analytic functions $f$ on the open unit disc $\mathbb{D}$ such that

$$
\iint_{\mathbb{D}}\left|f^{\prime}(z)\right|^{2} \varphi_{\mu}(z) d A(z)<\infty .
$$

Here $d A$ denotes normalized Lebesgue area measure on $\mathbb{D}$, i.e., $d A(z)=$ $(1 / \pi) r d r d t$, where $z=r e^{i t}$.

Lemma 3.1. If $f \in D(\mu)$, then $f \in H^{2}$. In fact, if $\mu \neq 0$, then there is a constant $C=C(\mu)>0$ such that for any analytic function $f$ on $\mathbb{D}$

$$
\|f\|_{H^{2}}^{2} \leq C\left(|f(0)|^{2}+\iint_{\mathbb{D}}\left|f^{\prime}(z)\right|^{2} \varphi_{\mu}(z) d A(z)\right) .
$$

Proof. We may assume $\mu \neq 0$. Suppose $f$ is analytic on $\mathbb{D}$. In $\S 2$ we noted that the $H^{2}$-norm of a function $f$ is equivalent to

$$
|f(0)|^{2}+\iint_{\mathbb{D}}\left|f^{\prime}(z)\right|^{2}\left(1-|z|^{2}\right) d A(z) ;
$$

thus there is a $c>0$ such that

$$
\|f\|_{H^{2}}^{2} \leq c\left(|f(0)|^{2}+\iint_{\mathbb{D}}\left|f^{\prime}(z)\right|^{2}\left(1-|z|^{2}\right) d A(z)\right) .
$$

If $z \in \mathbb{D}$ then

$$
\frac{1}{2 \pi} \int \frac{1}{\left|e^{i t}-z\right|^{2}} d \mu(t) \geq \frac{\mu(\mathbb{T})}{8 \pi} .
$$

In the following estimate, as in the rest of the paper, we shall use the letter $c$ to denote a generic constant whose value may change from line to line, but which never depends on the function $f$. We have

$$
\begin{aligned}
\|f\|_{H^{2}}^{2} & \leq c\left(|f(0)|^{2}+\iint_{\mathbb{D}}\left|f^{\prime}(z)\right|^{2}\left(1-|z|^{2}\right) d A(z)\right) \\
& \leq c\left(|f(0)|^{2}+\frac{8 \pi}{\mu(\mathbb{T})} \iint_{\mathbb{D}}\left|f^{\prime}(z)\right|^{2} \varphi_{\mu}(z) d A(z)\right) \\
& \leq c\left(|f(0)|^{2}+\iint_{\mathbb{D}}\left|f^{\prime}(z)\right|^{2} \varphi_{\mu}(z) d A(z)\right) \cdot
\end{aligned}
$$

It follows from Lemma 3.1 that we can define a norm on $D(\mu)$ by setting

$$
\|f\|_{\mu}^{2}=\|f\|_{H^{2}}^{2}+\iint_{\mathbb{D}}\left|f^{\prime}(z)\right|^{2} \varphi_{\mu}(z) d A(z) .
$$

We call attention to the special case when $\mu$ coincides with $m$, the Lebesgue measure on $\mathbb{T}$. In this case we have $D(m)=D$, the Dirichlet space. 
In the following example we shall see that even if $\mu$ is mutually absolutely continuous with respect to Lebesgue measure, it does not follow that $D(\mu) \subseteq H^{p}$ for any $p>2$.

Example 3.2. Consider the measure $\mu$ defined by $d \mu=\left|1-e^{i t}\right|^{2} d t=$ $2\left(1-\operatorname{Re} e^{i t}\right) d t$. We have for $z \in \mathbb{D}$,

$$
\begin{aligned}
\varphi_{\mu}(z) & =2(1-\operatorname{Re} z)=|1-z|^{2}+1-|z|^{2} \\
& \leq|1-z|^{2}+2|1-z| \leq 4|1-z| .
\end{aligned}
$$

Now let $0<\alpha<1 / 2$ and define $f(z)=(1-z)^{-\alpha}$. Then $f^{\prime}(z)=$ $-\alpha(1-z)^{-\alpha-1}$ and

$$
\iint_{\mathbb{D}}\left|f^{\prime}(z)\right|^{2} \varphi_{\mu}(z) d A(z) \leq 4 \alpha^{2} \iint_{\mathbb{D}}|(1-z)|^{-2 \alpha-1} d A(z)<\infty,
$$

since $2 \alpha+1<2$ and area measure integrates over singularities of this type. Thus, $f \in D(\mu)$, whenever $\alpha<1 / 2$.

On the other hand $f \in H^{p}$ if and only if $\alpha<1 / p$. Hence $D(\mu)$ is not contained in $H^{p}$ for any $p>2$.

Point evaluations on $D(\mu)$ are continuous because $D(\mu)$ is continuously contained in $H^{2}$ and point evaluations on $H^{2}$ are continuous.

Note that $D(\mu) \cap H^{\infty}$ is an algebra for every $\mu$. Indeed, if $f, g \in D(\mu) \cap$ $H^{\infty}$, then clearly $f g \in H^{\infty}$. A simple estimate shows that $f g \in D(\mu)$ as well.

We further note that since $\varphi_{\mu}(z) d A(z)$ is a finite measure on $\mathbb{D}, D(\mu)$ contains every $H^{2}$ function that has a bounded derivative. In particular, $D(\mu)$ contains the polynomials. We shall see later (Corollary 3.8) that the polynomials always form a dense set in $D(\mu)$.

Our first goal is to show that the linear transformation $M_{z}, f \rightarrow z f$, defines a bounded linear operator on $D(\mu)$. In addition, we shall see that $M_{z}$ is a cyclic 2 -isometry. Prior to this we need a few lemmas.

If $f$ is an analytic function on the unit disc, then $f$ has a Taylor expansion $f(z)=\sum_{n=0}^{\infty} \hat{f}(n) z^{n}$. Associated with $f$ and a nonnegative integer $k$ we define the truncated analytic function $T_{k} f$ by

$$
\left(T_{k} f\right)(z)=\sum_{n=k}^{\infty} \hat{f}(n) z^{n} .
$$

Furthermore, for $0<r<1$ we set $f_{r}(z)=f(r z)$.

In the following let $R \mathbb{D}$ denote the open disc of radius $R$. Then for any analytic function $f$ on $\mathbb{D}$ and any $0<R<1, \iint_{R \mathbb{D}}\left|f^{\prime}(z)\right|^{2} \varphi_{\mu}(z) d A(z)<\infty$, and if $f \in D(\mu)$, then $\|f\|_{H^{2}}^{2}+\iint_{R \mathbb{D}}\left|f^{\prime}(z)\right|^{2} \varphi_{\mu}(z) d A(z) \rightarrow\|f\|_{\mu}^{2}$ as $R \rightarrow 1$, $R<1$. It follows that an arbitrary $H^{2}$ function $f$ is in $D(\mu)$ if and only if $\iint_{R \mathbb{D}}\left|f^{\prime}(z)\right|^{2} \varphi_{\mu}(z) d A(z)$ is bounded independently of $R<1$. 
Lemma 3.3. Let $0<R<1$. If $f$ is analytic on $\mathbb{D}$, then

$$
\iint_{R \mathbb{D}}\left|f^{\prime}(z)\right|^{2} \varphi_{\mu}(z) d A(z)=\sum_{k=1}^{\infty} \frac{1}{2 \pi} \int_{0}^{2 \pi}\left|\left(T_{k} f\right)\left(R e^{i t}\right)\right|^{2} \varphi_{\mu}\left(R e^{i t}\right) d t .
$$

Proof. In the proof it will be convenient to use the least harmonic majorants of the subharmonic functions $\left|T_{k} f_{R}\right|^{2}$, so for $0<R<1$ and $k \geq 0$ define

$$
u_{k, R}(f)(z)=\frac{1}{2 \pi} \int_{0}^{2 \pi}\left|\left(T_{k} f_{R}\right)\left(e^{i s}\right)\right|^{2} P\left(z, e^{i s}\right) d s .
$$

Using Fubini's theorem and the fact that $P\left(R e^{i s}, e^{i t}\right)=P\left(R e^{i t}, e^{i s}\right)$ we see that for each nonnegative integer $k$,

$$
\begin{aligned}
& \frac{1}{2 \pi} \int_{0}^{2 \pi}\left|\left(T_{k} f\right)\left(\operatorname{Re}^{i s}\right)\right|^{2} \varphi_{\mu}\left(R e^{i s}\right) d s \\
& \quad=\frac{1}{2 \pi} \int_{0}^{2 \pi} \frac{1}{2 \pi} \int_{0}^{2 \pi}\left|\left(T_{k} f\right)\left(\operatorname{Re}^{i s}\right)\right|^{2} P\left(R e^{i t}, e^{i s}\right) d s d \mu(t) \\
& \quad=\frac{1}{2 \pi} \int_{0}^{2 \pi} u_{k, R}(f)\left(\operatorname{Re}^{i t}\right) d \mu(t) .
\end{aligned}
$$

Thus we have to show that

$$
\iint_{R \mathbb{D}}\left|f^{\prime}(z)\right|^{2} \varphi_{\mu}(z) d A(z)=\sum_{k=1}^{\infty} \frac{1}{2 \pi} \int_{0}^{2 \pi} u_{k, R}(f)\left(R e^{i t}\right) d \mu(t) .
$$

A second application of Fubini's theorem yields

$$
\iint_{R \mathbb{D}}\left|f^{\prime}(z)\right|^{2} \varphi_{\mu}(z) d A(z)=\frac{1}{2 \pi} \int_{0}^{2 \pi} \iint_{R \mathbb{D}}\left|f^{\prime}(z)\right|^{2} P\left(z, e^{i t}\right) d A(z) d \mu(t),
$$

hence it suffices to show that for each fixed $t \in[0,2 \pi]$ we have

$$
\iint_{R \mathbb{D}}\left|f^{\prime}(z)\right|^{2} P\left(z, e^{i t}\right) d A(z)=\sum_{k=1}^{\infty} u_{k, R}(f)\left(R e^{i t}\right) .
$$

To this end let $t \in[0,2 \pi]$. Two short computations with Poisson kernels show that for all nonnegative integers $n, m$,

$$
\frac{1}{2 \pi} \int_{0}^{2 \pi} e^{i(n-m) s} P\left(\operatorname{Re}^{i t}, e^{i s}\right) d s=R^{|n-m|} e^{i(n-m) t},
$$

and

$$
\begin{aligned}
\iint_{R \mathbb{D}} z^{n} \overline{z^{m}} P\left(z, e^{i t}\right) d A(z) & =2 \int_{0}^{R}\left(\frac{1}{2 \pi} \int_{0}^{2 \pi} e^{i(n-m) s} P\left(r e^{i s}, e^{i t}\right) d s\right) r^{n+m+1} d r \\
& =2 \int_{0}^{R}\left(r^{|n-m|} e^{i(n-m) t}\right) r^{n+m+1} d r \\
& =e^{i(n-m) t} \frac{\left(R^{2}\right)^{\max (n, m)+1}}{\max (n, m)+1}
\end{aligned}
$$


The Taylor series of $f(z)$ and $f^{\prime}(z)$ converge absolutely and uniformly on $R \mathbb{D}$; thus

$$
\begin{aligned}
& \iint_{R \mathbb{D}}\left|f^{\prime}(z)\right|^{2} P\left(z, e^{i t}\right) d A(z) \\
& =\sum_{n, m \geq 0} \sum_{m+1)}(n+n+1)(m+1) \overline{\hat{f}(m+1)} \iint_{R \mathbb{D}} z^{n} \overline{z^{m}} P\left(z, e^{i t}\right) d A(z)
\end{aligned}
$$

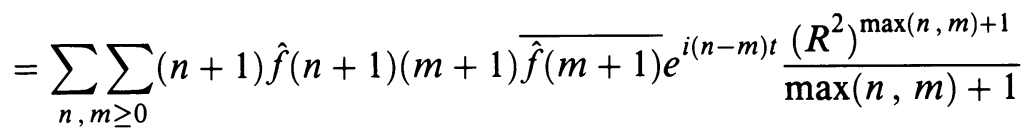

$$
\begin{aligned}
& =\sum_{n, m \geq 1} \sum_{n} \hat{f}(n) m \overline{\hat{f}(m)} e^{i(n-m) t} \frac{\left(R^{2}\right)^{\max (n, m)}}{\max (n, m)}
\end{aligned}
$$

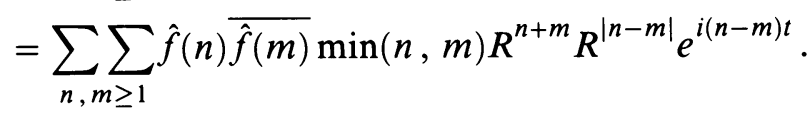

Further, for $k \geq 1$,

$$
\begin{aligned}
u_{k, R}(f)\left(R e^{i t}\right) & =\frac{1}{2 \pi} \int_{0}^{2 \pi}\left|\left(T_{k} f\right)\left(R e^{i s}\right)\right|^{2} P\left(R e^{i t}, e^{i s}\right) d s \\
& =\sum_{n, m \geq k} \hat{f}(n) \overline{\hat{f}(m)} R^{n+m} \frac{1}{2 \pi} \int_{0}^{2 \pi} e^{i(n-m) s} P\left(\operatorname{Re}^{i t}, e^{i s}\right) d s \\
& =\sum_{n, m \geq k} \sum_{\hat{f}(n) \overline{\hat{f}(m)} R^{n+m} R^{|n-m|} e^{i(n-m) t} .}
\end{aligned}
$$

Consequently, a change of summation yields

$$
\begin{aligned}
& \sum_{k=1}^{\infty} u_{k, R}(f)\left(R e^{i t}\right)=\sum_{k=1}^{\infty} \sum_{n, m \geq k} \sum_{\hat{f}}(n) \overline{\hat{f}(m)} R^{n+m} R^{|n-m|} e^{i(n-m) t} \\
& =\sum_{n, m \geq 1} \sum_{k=1}^{\min (n, m)} f(n) \overline{\hat{f}(m)} R^{n+m} R^{|n-m|} e^{i(n-m) t} \\
& =\sum_{n,} \sum_{m \geq 1} f(n) \overline{\hat{f}(m)} \min (n, m) R^{n+m} R^{|n-m|} e^{i(n-m) t} \\
& =\iint_{R \mathbb{D}}\left|f^{\prime}(z)\right|^{2} P\left(z, e^{i t}\right) d A(z) \text {. }
\end{aligned}
$$

Lemma 3.4. Let $0<R<1$. If $f$ is analytic on $\mathbb{D}$, then

$$
\begin{gathered}
\iint_{R \mathbb{D}}\left|(z f)^{\prime}(z)\right|^{2} \varphi_{\mu}(z) d A(z)-R^{2} \iint_{R \mathbb{D}}\left|f^{\prime}(z)\right|^{2} \varphi_{\mu}(z) d A(z) \\
=\frac{R^{2}}{2 \pi} \int_{0}^{2 \pi}\left|f\left(R e^{i t}\right)\right|^{2} \varphi_{\mu}\left(R e^{i t}\right) d t .
\end{gathered}
$$

Proof. We note that for all $k \geq 1$,

$$
\left(T_{k}(z f)\right)\left(\operatorname{Re}^{i t}\right)=\operatorname{Re}^{i t}\left(T_{k-1} f\right)\left(\operatorname{Re}^{i t}\right),
$$


and hence $\left|\left(T_{k}(z f)\right)\left(\operatorname{Re}^{i t}\right)\right|^{2}=R^{2}\left|\left(T_{k-1} f\right)\left(R e^{i t}\right)\right|^{2}$. Now we apply Lemma 3.3 to the functions $f$ and $z f$ :

$$
\begin{aligned}
& \iint_{R \mathbf{D}}\left|(z f)^{\prime}(z)\right|^{2} \varphi_{\mu}(z) d A(z) \\
&=\sum_{k=1}^{\infty} \frac{1}{2 \pi} \int_{0}^{2 \pi}\left|\left(T_{k}(z f)\right)\left(\operatorname{Re}^{i t}\right)\right|^{2} \varphi_{\mu}\left(\operatorname{Re}^{i t}\right) d t \\
&=R^{2} \sum_{k=1}^{\infty} \frac{1}{2 \pi} \int_{0}^{2 \pi}\left|\left(T_{k-1} f\right)\left(\operatorname{Re}^{i t}\right)\right|^{2} \varphi_{\mu}\left(\operatorname{Re} e^{i t}\right) d t \\
&=\frac{R^{2}}{2 \pi} \int_{0}^{2 \pi}\left|f\left(\operatorname{Re}^{i t}\right)\right|^{2} \varphi_{\mu}\left(\operatorname{Re}^{i t}\right) d t \\
& \quad+R^{2} \sum_{k=1}^{\infty} \frac{1}{2 \pi} \int_{0}^{2 \pi}\left|\left(T_{k} f\right)\left(\operatorname{Re}^{i t}\right)\right|^{2} \varphi_{\mu}\left(\operatorname{Re}^{i t}\right) d t \\
&= \frac{R^{2}}{2 \pi} \int_{0}^{2 \pi}\left|f\left(\operatorname{Re}^{i t}\right)\right|^{2} \varphi_{\mu}\left(\operatorname{Re}^{i t}\right) d t+R^{2} \iint_{R \mathbb{D}}\left|f^{\prime}(z)\right|^{2} \varphi_{\mu}(z) d A(z) .
\end{aligned}
$$

This concludes the proof of Lemma 3.4.

Prior to proving that $M_{z}$ is a bounded operator on $D(\mu)$ we need one more lemma.

Lemma 3.5. There is a constant $c=c(\mu)$ such that for every $f \in D(\mu)$ and $0<R<1$,

$$
\frac{1}{2 \pi} \int_{0}^{2 \pi}\left|f\left(\operatorname{Re}^{i t}\right)\right|^{2} \varphi_{\mu}\left(R e^{i t}\right) d t \leq c\|f\|_{\mu}^{2} .
$$

Proof. We have $f\left(\operatorname{Re}^{i t}\right)=f(0)+\left(T_{1} f\right)\left(R e^{i t}\right)$, hence

$$
\begin{aligned}
\left|f\left(R e^{i t}\right)\right|^{2} & \leq 2\left(|f(0)|^{2}+\left|\left(T_{1} f\right)\left(R e^{i t}\right)\right|^{2}\right) \\
& \leq 2\left(\|f\|_{H^{2}}^{2}+\sum_{k=1}^{\infty}\left|\left(T_{k} f\right)\left(R e^{i t}\right)\right|^{2}\right) .
\end{aligned}
$$

By Lemma 3.3 this implies

$$
\begin{aligned}
& \frac{1}{2 \pi} \int_{0}^{2 \pi}\left|f\left(\operatorname{Re}^{i t}\right)\right|^{2} \varphi_{\mu}\left(R e^{i t}\right) d t \\
& \quad \leq \frac{1}{\pi}\|f\|_{H^{2}}^{2} \int_{0}^{2 \pi} \varphi_{\mu}\left(R e^{i t}\right) d t+2 \iint_{R \mathbb{D}}\left|f^{\prime}(z)\right|^{2} \varphi_{\mu}(z) d A(z) \\
& \quad \leq \frac{1}{\pi}\|f\|_{H^{2}}^{2} \mu(\mathbb{T})+2 \iint_{\mathbb{D}}\left|f^{\prime}(z)\right|^{2} \varphi_{\mu}(z) d A(z) \\
& \quad \leq c\|f\|_{\mu}^{2} \text {. }
\end{aligned}
$$

Theorem 3.6. Let $f$ be analytic on $\mathbb{D}$. Then

$$
f \in D(\mu) \Leftrightarrow z f \in D(\mu) \text {. }
$$


In fact, one has $\|f\|_{\mu}^{2} \leq\|z f\|_{\mu}^{2} \leq(c+1)\|f\|_{\mu}^{2}$, where $c$ is the constant from Lemma 3.5.

Consequently, multiplication by $z$ defines a bounded linear operator on $D(\mu)$. We shall denote it by $\left(M_{z}, D(\mu)\right)$ or simply by $M_{z}$.

Proof. First assume that $f \in D(\mu)$. Then both $f$ and $z f$ are $H^{2}$ functions, and for $0<R<1$ we have by Lemmas 3.4 and 3.5

$$
\begin{aligned}
& \|z f\|_{H^{2}}^{2}+\iint_{R \mathbb{D}}\left|(z f)^{\prime}(z)\right|^{2} \varphi_{\mu}(z) d A(z) \\
& \quad=\|f\|_{H^{2}}^{2}+\frac{R^{2}}{2 \pi} \int_{0}^{2 \pi}\left|f\left(\operatorname{Re}^{i t}\right)\right|^{2} \varphi_{\mu}\left(R e^{i t}\right) d t+R^{2} \iint_{R \mathbb{D}}\left|f^{\prime}(z)\right|^{2} \varphi_{\mu}(z) d A(z) \\
& \quad \leq(c+1)\|f\|_{\mu}^{2} .
\end{aligned}
$$

Letting $R \rightarrow 1$ we obtain $z f \in D(\mu)$ and $\|z f\|_{\mu}^{2} \leq(c+1)\|f\|_{\mu}^{2}$.

Now assume that $z f \in D(\mu)$. Again it follows that both $f$ and $z f$ are in $H^{2}$. For $0<R<1$ we have by Lemma 3.4

$$
\begin{aligned}
\|z f\|_{\mu}^{2} & \geq\|z f\|_{H^{2}}^{2}+\iint_{R \mathbb{D}}\left|(z f)^{\prime}(z)\right|^{2} \varphi_{\mu}(z) d A(z) \\
& =\|f\|_{H^{2}}^{2}+\frac{R^{2}}{2 \pi} \int_{0}^{2 \pi}\left|f\left(\operatorname{Re}^{i t}\right)\right|^{2} \varphi_{\mu}\left(R e^{i t}\right) d t+R^{2} \iint_{R \mathbb{D}}\left|f^{\prime}(z)\right|^{2} \varphi_{\mu}(z) d A(z) \\
& \geq\|f\|_{H^{2}}^{2}+R^{2} \iint_{R \mathbb{D}}\left|f^{\prime}(z)\right|^{2} \varphi_{\mu}(z) d A(z) .
\end{aligned}
$$

Now we let $R \rightarrow 1$ to obtain that $f \in D(\mu)$ and $\|z f\|_{\mu} \geq\|f\|_{\mu}$.

Theorem 3.7. $\left(M_{z}, D(\mu)\right)$ is a 2-isometry, i.e., for every $f \in D(\mu)$ we have

$$
\left\|z^{2} f\right\|_{\mu}^{2}-2\|z f\|_{\mu}^{2}+\|f\|_{\mu}^{2}=0 .
$$

Proof. Clearly, $\left\|z^{2} f\right\|_{H^{2}}^{2}-2\|z f\|_{H^{2}}^{2}+\|f\|_{H^{2}}^{2}=0$ for every $f \in D(\mu)$. Thus, it is enough to show that

$$
\iint_{R \mathbb{D}}\left(\left|\left(z^{2} f\right)^{\prime}(z)\right|^{2}-2\left|(z f)^{\prime}(z)\right|^{2}+\left|f^{\prime}(z)\right|^{2}\right) \varphi_{\mu}(z) d A(z) \rightarrow 0 \quad \text { as } R \rightarrow 1
$$

Consider

$$
\begin{aligned}
\iint_{R \mathbb{D}} & \left(\left|\left(z^{2} f\right)^{\prime}(z)\right|^{2}-2 R^{2}\left|(z f)^{\prime}(z)\right|^{2}+R^{4}\left|f^{\prime}(z)\right|^{2}\right) \varphi_{\mu}(z) d A(z) \\
= & \iint_{R \mathbb{D}}\left(\left|\left(z^{2} f\right)^{\prime}(z)\right|^{2}-R^{2}\left|(z f)^{\prime}(z)\right|^{2}\right) \varphi_{\mu}(z) d A(z) \\
& -R^{2} \iint_{R \mathbb{D}}\left(\left|(z f)^{\prime}(z)\right|^{2}-R^{2}\left|f^{\prime}(z)\right|^{2}\right) \varphi_{\mu}(z) d A(z) \\
= & \frac{R^{2}}{2 \pi} \int_{0}^{2 \pi}\left|R e^{i t} f\left(R e^{i t}\right)\right|^{2} \varphi_{\mu}\left(R e^{i t}\right) d t \\
& -R^{2}\left(\frac{R^{2}}{2 \pi} \int_{0}^{2 \pi}\left|f\left(\operatorname{Re}^{i t}\right)\right|^{2} \varphi_{\mu}\left(R e^{i t}\right) d t\right) \\
= & 0,
\end{aligned}
$$

by Lemma 3.4 applied to $z f$ and $f$. 
Hence

$$
\begin{aligned}
\iint_{R \mathbb{D}}\left(\left|\left(z^{2} f\right)^{\prime}(z)\right|^{2}-2\left|(z f)^{\prime}(z)\right|^{2}+\left|f^{\prime}(z)\right|^{2}\right) \varphi_{\mu}(z) d A(z) \\
=-2\left(1-R^{2}\right) \iint_{R \mathbb{D}}\left|(z f)^{\prime}(z)\right|^{2} \varphi_{\mu}(z) d A(z) \\
\quad+\left(1-R^{4}\right) \iint_{R \mathbb{D}}\left|f^{\prime}(z)\right|^{2} \varphi_{\mu}(z) d A(z) \\
\quad \rightarrow 0 \quad \text { as } R \rightarrow 1, \text { as required. }
\end{aligned}
$$

In the following corollary we list some immediate consequences of Theorems 3.6 and 3.7. We keep the finite positive Borel measure fixed and write $M_{z}$ instead of $\left(M_{z}, D(\mu)\right)$. The direct difference of two subspaces $\mathscr{M}$ and $\mathscr{N}$ is $\mathscr{M} \ominus \mathscr{N}=\mathscr{M} \cap \mathscr{N}^{\perp}$, and we let $\mathscr{B}_{1}(\mathbb{D})$ denote the Cowen-Douglas class of operators (for a definition see [5]).

Corollary 3.8. (a) If $\lambda \in \mathbb{D}$, then $M_{z}-\lambda$ is bounded below and $\operatorname{dim} D(\mu) \ominus$ $(z-\lambda) D(\mu)=\operatorname{dim} \operatorname{ker}\left(M_{z}-\lambda\right)^{*}=1$.

(b) $\sigma\left(M_{z}\right)=\mathbb{D}^{-}$.

(c) $M_{z}^{*} \in \mathscr{B}_{1}(\mathbb{D})$.

(d) The polynomials are dense in $D(\mu)$.

Proof. (a) Let $\lambda \in \mathbb{D}$ and $f \in D(\mu)$, then $\left\|\left(M_{z}-\lambda\right) f\right\|_{\mu} \geq\|z f\|_{\mu}-|\lambda|\|f\|_{\mu} \geq$ $(1-|\lambda|)\|f\|_{\mu}$, because by Theorem $3.6 M_{z}$ is bounded below by 1 .

It now follows from Theorem 3.6 above and Lemma 2.1 of [8] that

$$
\operatorname{dim} D(\mu) \ominus(z-\lambda) D(\mu)=\operatorname{dim} \operatorname{ker}\left(M_{z}-\lambda\right)^{*}=1 .
$$

(b) By (a) we have $\mathbb{D}^{-} \subseteq \sigma\left(M_{z}\right)$. Furthermore, Lemma 1(c) of [9] states that for 2-isometries we always have $\sigma\left(M_{z}\right) \subseteq \mathbb{D}^{-}$.

(c) follows from Theorem 2.10 of [8].

(d) Note that the constant functions are orthogonal to $z D(\mu)$, thus by (a) we have $D(\mu) \ominus z D(\mu)=\{f: \mathbb{D} \rightarrow \mathbb{C}: f$ is constant $\}$. Now (d) follows from Theorem 1 of [9].

\section{Boundary VALUES}

In [9] we saw that, if $T$ is a 2-isometry, then the positive operator $D$, defined by $D^{2}=T^{*} T-I$, plays an important role in the study of $T$. We shall now see that for the 2-isometry $\left(M_{z}, D(\mu)\right)$ the operator $D$ is unitarily equivalent to an operator $B$ that maps $D(\mu)$ into $L^{2}(\mu)$. Thus, associated with every $f \in D(\mu)$ one has a "boundary value function" $\tilde{f} \in L^{2}(\mu)$. The results of this section will be important in what follows.

Let $P^{2}(\mu)$ denote the closure of the polynomials in $L^{2}(\mu)$. The norm on $P^{2}(\mu)$ is given by

$$
\|f\|_{P^{2}(\mu)}^{2}=\frac{1}{2 \pi} \int_{0}^{2 \pi}\left|f\left(e^{i s}\right)\right|^{2} d \mu(s)
$$


Theorem 4.1. There is a bounded linear transformation $B$ from $D(\mu)$ into $P^{2}(\mu)$ which satisfies:

(a) If $f \in D(\mu)$ and the Taylor series of $f$ converges absolutely and uniformly on $\mathbb{D}^{-}$, then $(B f)\left(e^{i t}\right)=f\left(e^{i t}\right)$ a.e. $[\mu]$.

(b) For all $f \in D(\mu)$ we have

$$
\begin{aligned}
\|z f\|_{\mu}^{2}-\|f\|_{\mu}^{2} & =\lim _{R \rightarrow 1} \frac{1}{2 \pi} \int_{0}^{2 \pi}\left|f\left(\operatorname{Re}^{i t}\right)\right|^{2} \varphi_{\mu}\left(\operatorname{Re}^{i t}\right) d t \\
& =\frac{1}{2 \pi} \int_{0}^{2 \pi}\left|(B f)\left(e^{i s}\right)\right|^{2} d \mu(s)=\|B f\|_{P^{2}(\mu)}^{2} .
\end{aligned}
$$

(c) If $p$ is a polynomial and $f \in D(\mu)$, then $B(p f)\left(e^{i t}\right)=p\left(e^{i t}\right)(B f)\left(e^{i t}\right)$ a.e. $[\mu]$.

(d) If $p$ is a polynomial, then

$$
\|p\|_{\mu}^{2}=\|p\|_{H^{2}}^{2}+\sum_{k=1} \frac{1}{2 \pi} \int_{0}^{2 \pi}\left|\left(T_{k} p\right)\left(e^{i t}\right)\right|^{2} d \mu(t) .
$$

Proof. If $f \in D(\mu)$ such that the Taylor series of $f$ converges absolutely and uniformly on $\mathbb{D}^{-}$, then define $B f$ by $(B f)\left(e^{i t}\right)=f\left(e^{i t}\right)$.

The polynomials are dense in $D(\mu)$; thus, in order to verify that $B$ extends to a continuous operator on all of $D(\mu)$ we only have to prove that (b) holds for all $f$ as above. We shall show that

$$
\|z f\|_{\mu}^{2}-\|f\|_{\mu}^{2}=\frac{1}{2 \pi} \int_{0}^{2 \pi}\left|f\left(e^{i s}\right)\right|^{2} d \mu(s)
$$

whenever the Taylor series of $f$ converges absolutely and uniformly on $\mathbb{D}^{-}$. This will imply both parts (a) and (b) of the theorem because by definition of the $D(\mu)$ norm and Lemma 3.4 we have

$$
\|z f\|_{\mu}^{2}-\|f\|_{\mu}^{2}=\lim _{R \rightarrow 1} \frac{1}{2 \pi} \int_{0}^{2 \pi}\left|f\left(R e^{i t}\right)\right|^{2} \varphi_{\mu}\left(R e^{i t}\right) d t .
$$

Let $f \in D(\mu)$ be such that the Taylor series of $f$ converges absolutely and uniformly on $\mathbb{D}^{-}$. As in the proof of Lemma 3.3, an application of Fubini's theorem and a computation with the Poisson kernel yields

$$
\begin{aligned}
\|z f\|_{\mu}^{2}-\|f\|_{\mu}^{2} & =\lim _{R \rightarrow 1} \frac{1}{2 \pi} \int_{0}^{2 \pi} \frac{1}{2 \pi} \int_{0}^{2 \pi}\left|f\left(\operatorname{Re}^{i t}\right)\right|^{2} P\left(R e^{i t}, e^{i s}\right) d t d \mu(s) \\
& =\lim _{R \rightarrow 1} \frac{1}{2 \pi} \int_{0}^{2 \pi} \sum_{n, m \geq 0} \sum_{m} \hat{f}(n) \overline{\hat{f}(m)} R^{n+m} R^{|n-m|} e^{i(n-m) s} d \mu(s) \\
& =\frac{1}{2 \pi} \int_{0}^{2 \pi}\left|f\left(e^{i s}\right)\right|^{2} d \mu(s)
\end{aligned}
$$

by the Dominated Convergence Theorem.

To verify (c) let $p$ be a polynomial and $f \in D(\mu)$. We have to show that $B(p f)\left(e^{i t}\right)=p\left(e^{i t}\right)(B f)\left(e^{i t}\right)$ a.e. $[\mu]$. To this end, let $\varepsilon>0$ and choose a 
polynomial $q$ such that $\|f-q\|_{\mu}^{2}<\varepsilon$. We note that $B(p q)\left(e^{i t}\right)=p q\left(e^{i t}\right)=$ $p\left(e^{i t}\right) B q\left(e^{i t}\right)$ a.e. $[\mu]$ by $(\mathrm{a})$, thus

$$
\begin{aligned}
\| B(p f) & -p B f\left\|_{P^{2}(\mu)}^{2} \leq\right\| B(p f)-B(p q)\left\|_{P^{2}(\mu)}^{2}+\right\| p(B q-B f) \|_{P^{2}(\mu)}^{2} \\
\leq & \left(\|z p(f-q)\|_{\mu}^{2}-\|p(f-q)\|_{\mu}^{2}\right) \\
& +\frac{1}{2 \pi} \int_{0}^{2 \pi}\left|p\left(e^{i s}\right)\right|^{2}\left|(B q-B f)\left(e^{i s}\right)\right|^{2} d \mu(s) .
\end{aligned}
$$

Multiplication by $p$ and by $z p$ are bounded linear operators on $D(\mu)$, hence we obtain

$$
\begin{aligned}
\|B(p f)-p B f\|_{P^{2}(\mu)}^{2} & \leq C_{1}(p)\|f-q\|_{\mu}^{2}+\|p\|_{\infty}^{2}\|B q-B f\|_{P^{2}(\mu)}^{2} \\
& \leq C_{2}(p)\|f-q\|_{\mu}^{2} \leq C_{2}(p) \varepsilon .
\end{aligned}
$$

Thus $B(p f)=p B f$ since $\varepsilon$ was arbitrary.

Finally, we shall show that (d) holds. Let $p$ be a polynomial. Using the definition of the $D(\mu)$ norm and Lemma 3.3 we have to show that

$$
\sum_{k=1} \frac{1}{2 \pi} \int_{0}^{2 \pi}\left|\left(T_{k} p\right)\left(e^{i t}\right)\right|^{2} d \mu(t)=\sum_{k=1} \lim _{R \rightarrow 1} \frac{1}{2 \pi} \int_{0}^{2 \pi}\left|\left(T_{k} p\right)\left(R e^{i t}\right)\right|^{2} \varphi_{\mu}\left(R e^{i t}\right) d t .
$$

The sums are finite since $p$ is a polynomial; thus the interchange of sum and limit causes no problems.

This identity follows immediately from an application of $(b)$ to the functions $T_{k} p$.

Note that the map $B$ may have a nontrivial kernel; e.g. let $\mu$ be a unit point mass at 1 and $f$ be any polynomial vanishing at 1 , then $(B f)\left(e^{i t}\right)=0$ a.e. $[\mu]$.

Of course $B$ depends on the measure $\mu$. We shall write $B=B^{\mu}$ when we want to stress this dependence. It may happen that an analytic function $f$ is contained in $D(\mu) \cap D(\nu)$ for two measures $\mu$ and $\nu$. We shall see below that in this case there is a Borel measurable function $\tilde{f}$ on $\mathbb{T}$ such that $\left(B^{\mu} f\right)\left(e^{i t}\right)=\tilde{f}\left(e^{i t}\right)$ a.e. $[\mu]$ and $\left(B^{\nu} f\right)\left(e^{i t}\right)=\tilde{f}\left(e^{i t}\right)$ a.e. $[\nu]$.

Furthermore, the spaces $D(\mu)$ are contained in $H^{2}$; hence associated with every $f \in D(\mu)$ there is a radial limit function $\hat{f} \in L^{2}(m)$ defined a.e. by $\hat{f}\left(e^{i t}\right)=\lim _{r \rightarrow 1} f\left(r e^{i t}\right)$. If the Taylor series of $f$ converges absolutely and uniformly on $\mathbb{D}^{-}$, then $\hat{f}\left(e^{i t}\right)=f\left(e^{i t}\right)=B^{\mu} f\left(e^{i t}\right)$ a.e. $[\mu]$. However, in general the measure $\mu$ may not be comparable with Lebesgue measure on $\mathbb{T}$; thus for general functions $f \in D(\mu)$ we have to distinguish between $\hat{f}$ and $B^{\mu} f$. The next proposition will imply that if $\mu$ is absolutely continuous, then $\hat{f}\left(e^{i t}\right)=B^{\mu} f\left(e^{i t}\right)$ a.e. $[\mu]$.

In the following we shall write $\mu \ll \nu$, if $\mu$ is absolutely continuous with respect to $\nu$. We shall write $[\mu]=[\nu]$, if $\mu$ and $\nu$ are mutually absolutely continuous. 
Lemma 4.2. Let $\mu$ and $\nu$ be positive finite Borel measures on $\mathbb{T}$. If $\mu \ll \nu$ and $f \in D(\mu) \cap D(\nu)$, then $B^{\mu} f\left(e^{i t}\right)=B^{\nu} f\left(e^{i t}\right)$ a.e. $[\mu]$.

Proof. Define a finite positive Borel measure $\sigma$ on $\mathbb{T}$ by $d \sigma=d \mu+d \nu$. Then $\mu \ll \nu \ll \sigma . f \in D(\mu) \cap D(\nu)$ implies that $f \in D(\sigma)$.

By Corollary 3.8(d) there is a sequence of polynomials $\left\{p_{k}\right\}$ such that $\left\{p_{k}\right\} \rightarrow$ $f$ in $D(\sigma)$. It follows from the definition of $\sigma$ that $\left\{p_{k}\right\} \rightarrow f$ in $D(\mu)$ and in $D(\nu)$. Thus, Theorem 4.1 implies that $\left\{B^{\mu} p_{k}\right\} \rightarrow B^{\mu} f$ in $P^{2}(\mu)$ and $\left\{B^{\nu} p_{k}\right\} \rightarrow B^{\nu} f$ in $P^{2}(\nu)$. Also by Theorem 4.1 we have $B^{\mu} p_{k}\left(e^{i t}\right)=p_{k}\left(e^{i t}\right)$ a.e. $[\mu]$ and $B^{\nu} p_{k}\left(e^{i t}\right)=p_{k}\left(e^{i t}\right)$ a.e. $[\nu]$. Hence we can first choose a subsequence of $\left\{p_{k}\left(e^{i t}\right)\right\}$ that converges a.e. $[\mu]$ to $B^{\mu} f\left(e^{i t}\right)$ and then choose a further subsequence, which converges a.e. $[\nu]$ to $B^{\nu} f\left(e^{i t}\right)$. We denote this subsequence by $\left\{q_{k}\right\}$. The assumption $\mu \ll \nu$ implies that $\left\{q_{k}\left(e^{i t}\right)\right\} \rightarrow B^{\nu} f\left(e^{i t}\right)$ a.e. $[\mu]$, as well as $\left\{q_{k}\left(e^{i t}\right)\right\} \rightarrow B^{\mu} f\left(e^{i t}\right)$ a.e. $[\mu]$. Thus $B^{\nu} f\left(e^{i t}\right)=B^{\mu} f\left(e^{i t}\right)$ a.e. $[\mu]$.

Proposition 4.3. Let $\mathscr{S}$ be a collection of positive finite Borel measures on $\mathbb{T}$ such that there is a countable subcollection $\left\{\mu_{n}\right\}_{n \in \mathbb{N}} \subseteq \mathscr{S}$ such that $\forall \nu \in \mathscr{S} \exists n \in$ $\mathbb{N} \ni \nu \ll \mu_{n}$.

For every measure $\nu \in \mathscr{S}$ let $d \nu=d \nu_{a}+d \nu_{s}$ be the decomposition into absolutely continuous and singular parts.

If $f \in \bigcap_{\nu \in S} D(\nu)$, then there is a Borel measurable function $\tilde{f}$ such that for every $\nu \in \mathscr{S} \tilde{f}\left(e^{i t}\right)=B^{\nu} f\left(e^{i t}\right)$ a.e. [ $[\nu]$ and $\tilde{f}\left(e^{i t}\right)=\hat{f}\left(e^{i t}\right)$ a.e. $\left[\nu_{a}\right]$.

In particular, if $f \in H^{2}$ and if $\mathscr{S}_{f}$ consists of all positive finite Borel measures $\nu$ that are absolutely continuous with respect to Lebesgue measure and such that $f \in D(\nu)$, then for every $\nu \in \mathscr{S}_{f}$ we have $B^{\nu} f\left(e^{i t}\right)=\hat{f}\left(e^{i t}\right)$ a.e. $[\nu]$.

Proof. Let $\mathscr{S}$ be a collection of measures satisfying the hypothesis of the proposition, and let $f \in \bigcap_{\nu \in \mathscr{S}} D(\nu)$.

We may assume that for every $n \in \mathbb{N}, \mu_{n} \neq 0$. Furthermore, by multiplying $\mu_{n}$ with a constant we even may assume that $\mu_{n}(\mathbb{T})=1$. Let $c_{n}=$ $\max \left\{\|f\|_{\mu_{n}}^{2}, 1\right\}$.

Now define a positive Borel measure $\sigma$ on $\mathbb{T}$ by $d \sigma=\sum_{n=1}^{\infty} \frac{1}{c_{n}} \frac{1}{2^{n}} d \mu_{n}$. Then $\sigma(\mathbb{T}) \leq 1$, i.e., $\sigma$ is a finite Borel measure, and $\mu_{n} \ll \sigma$. By the choice of $c_{n}$ one readily verifies that $f \in D(\sigma)$.

We set $\tilde{f}\left(e^{i t}\right)=\left(B^{\sigma} f\right)\left(e^{i t}\right)$ whenever this is defined and $\tilde{f}\left(e^{i t}\right)=0$ otherwise.

Let $\nu \in \mathscr{S}$. Then the assumption implies $\nu \ll \sigma$ and $f \in D(\sigma) \cap D(\nu)$; thus, it follows from Lemma 4.2 that $B^{\nu} f\left(e^{i t}\right)=B^{\sigma} f\left(e^{i t}\right)=\tilde{f}\left(e^{i t}\right)$ a.e. $[\nu]$.

To finish the proof we have to show that $\tilde{f}\left(e^{i t}\right)=\hat{f}\left(e^{i t}\right)$ a.e. $\left[\nu_{a}\right]$ for every $\nu \in \mathscr{S}$. The proof is similar to the proof of Lemma 4.2. Let $\left\{p_{n}\right\}$ be a sequence of polynomials that converges to $f$ in $D(\sigma)$.

Then $\left\{p_{n}\right\}$ converges to $f$ in $H^{2}$ and to $\tilde{f}$ in $P^{2}(\sigma)$. It follows that $\left\{\hat{p}_{n}\right\}$ 
converges to $\hat{f}$ in $L^{2}(m)$. Thus, as before there is a subsequence $\left\{p_{n_{k}}\right\}$ of $\left\{p_{n}\right\}$ such that $\left\{p_{n_{k}}\left(e^{i t}\right)\right\} \rightarrow \hat{f}\left(e^{i t}\right)$ a.e. $[m]$ and $\left\{p_{n_{k}}\left(e^{i t}\right)\right\} \rightarrow \tilde{f}\left(e^{i t}\right)$ a.e. $[\sigma]$.

The measure $\nu_{a}$ is absolutely continuous with respect to both $\sigma$ and Lebesgue measure; thus

$$
\left\{p_{n_{k}}\left(e^{i t}\right)\right\} \rightarrow \hat{f}\left(e^{i t}\right) \text { a.e. }\left[\nu_{a}\right]
$$

as well as

$$
\left\{p_{n_{k}}\left(e^{i t}\right)\right\} \rightarrow \tilde{f}\left(e^{i t}\right) \text { a.e. }\left[\nu_{a}\right] .
$$

This implies $\tilde{f}\left(e^{i t}\right)=\hat{f}\left(e^{i t}\right)$ a.e. $\left[\nu_{a}\right]$.

In the following we shall often have no need to refer to the map $B$; thus for ease of notation we shall write $\tilde{f}$ instead of $B f$. If in some context several measures are present, then by $\tilde{f}$ we mean the function whose existence is guaranteed by Proposition 4.3. However, we keep in mind that $\tilde{f}$ is associated with a family of measures as above.

\section{THE REPRESENTATION THEOREM FOR CYCLIC ANALYTIC TWO-ISOMETRIES}

We are now ready to prove our main result about the spaces $D(\mu)$.

Theorem 5.1. Let $T \in \mathscr{L}(\mathscr{H})$. The following are equivalent:

(a) $T$ is a cyclic analytic 2-isometry,

(b) $T$ is a 2-isometry and $T^{*} \in \mathscr{B}_{1}(\mathbb{D})$,

(c) there is a positive finite Borel measure $\mu$ on $\mathbb{T}$ such that $T$ is unitarily equivalent to $\left(M_{z}, D(\mu)\right)$.

Proof. In $\S 3$ we saw that (c) implies both (a) and (b). Furthermore, to see that (b) $\Rightarrow$ (a), recall from [5] that if $T^{*} \in \mathscr{B}_{1}(\mathbb{D})$, then $T$ is unitarily equivalent to $M_{z}$ acting on some Hilbert space of analytic functions, hence $\bigcap_{n \geq 0} T^{n} \mathscr{H}=$ $\{0\}$. It now follows from Theorem 1 of [9] that $T$ is cyclic.

Thus, to finish the proof of the theorem we have to show $(a) \Rightarrow(c)$. Assume that $T$ is a cyclic analytic 2-isometry. By Lemma 1 of [9] any 2-isometry is bounded below by 1 . Also, since $T$ is analytic it cannot be onto; thus that $T$ has a cyclic vector implies that $\operatorname{dim} \operatorname{ker} T^{*}=1$. So let $x_{0} \in \operatorname{ker} T^{*},\left\|x_{0}\right\|=1$. By Theorem 1 of [9] $x_{0}$ is a cyclic vector for $T$, and for any polynomial $p$ we have by Lemma 2.1

$$
\left\|p(T) x_{0}\right\|^{2}=\|p\|_{H^{2}}^{2}+\sum_{k=1}\left\|D p_{k}(T) x_{0}\right\|^{2}
$$

where $D^{2}=T^{*} T-I$ and $p_{k}(z)=\left(T_{k} p\right)(z)\left(T_{k}\right.$ as in $\left.\S 3\right)$.

Note that $\operatorname{ker} D \in \operatorname{Lat} T$. Indeed if $x \in \operatorname{ker} D$, then $\|D T x\|^{2}=\left\|T^{2} x\right\|^{2}-$ $\|T x\|^{2}=\|T x\|^{2}-\|x\|^{2}=\|D x\|^{2}=0$, since $T$ is a 2-isometry.

Define a norm on $(\operatorname{ker} D)^{\perp}$ by $\|x\|_{\mathscr{K}}=\|D x\|$, and let $\mathscr{K}$ be the completion of $\left((\operatorname{ker} D)^{\perp},\|\|_{\mathscr{K}}\right)$. Now define a linear transformation $\tilde{T}$ on $(\operatorname{ker} D)^{\perp}$ by $\widetilde{T} x=P T x$, where $P$ denotes the projection of $\mathscr{H}$ onto $(\operatorname{ker} D)^{\perp}$. For every 
$x \in(\operatorname{ker} D)^{\perp}$ we have $\|\tilde{T} x\|_{\mathscr{K}}=\|D P T x\|=\|D T x\|=\|D x\|=\|x\|_{\mathscr{K}}$. Thus, $\widetilde{T}$ extends to be an isometry on $\mathscr{K}$.

Claim. $P x_{0}$ is a cyclic vector for $\widetilde{T} \in \mathscr{L}(\mathscr{K})$.

Proof (of the claim). $\operatorname{Ker} D^{\perp}$ is dense in $\mathscr{K}$; thus it is enough to show that an arbitrary element $x \in \operatorname{ker} D^{\perp}$ can be approximated in \|\|$_{\mathscr{K}}$ by elements of the form $q(\widetilde{T}) P x_{0}$, where $q$ is a polynomial. But $q(\widetilde{T}) P x_{0}=P q(T) P x_{0}=$ $P q(T) x_{0}$, because $(I-P) x_{0} \in \operatorname{ker} D$, hence $q(T)(I-P) x_{0} \in \operatorname{ker} D=\operatorname{ker} P$. Thus

$$
\begin{aligned}
\left\|x-q(\widetilde{T}) P x_{0}\right\|_{\mathscr{K}} & =\left\|D x-D P q(T) x_{0}\right\|=\left\|D x-D q(T) x_{0}\right\| \\
& \leq\|D\|\left\|x-q(T) x_{0}\right\| .
\end{aligned}
$$

Now the claim follows, because $x_{0}$ is a cyclic vector for $T$.

We now know that $\widetilde{T}$ is a cyclic isometry; hence it is a cyclic subnormal operator. By Bram's theorem (see [2]) there is a positive finite Borel measure $\mu$ on $\sigma(T) \subseteq \mathbb{T}$ such that $\widetilde{T}$ is unitarily equivalent to $M_{z}$ on $P^{2}(\mu)$, and the cyclic vector $P x_{0}$ corresponds to the function 1. Thus $\left\|q(\tilde{T}) P x_{0}\right\|_{\mathscr{K}}^{2}=$ $\frac{1}{2 \pi} \int_{0}^{2 \pi}\left|q\left(e^{i t}\right)\right|^{2} d \mu(t)$ for every polynomial $q$. Hence, if $q$ is a polynomial, then

$$
\begin{aligned}
\left\|D q(T) x_{0}\right\|^{2} & =\left\|D q(T) P x_{0}\right\|^{2}=\left\|D P q(T) P x_{0}\right\|^{2} \\
& =\left\|q(\widetilde{T}) P x_{0}\right\|_{\mathscr{K}}^{2}=\frac{1}{2 \pi} \int_{0}^{2 \pi}\left|q\left(e^{i t}\right)\right|^{2} d \mu(t) .
\end{aligned}
$$

Define a linear transformation $U$ from the polynomials into $\mathscr{H}$ by $p \rightarrow$ $p(T) x_{0}$. The polynomials are dense in $D(\mu)$, thus the following computation will show that $U$ extends to be an isometry $D(\mu) \rightarrow \mathscr{H}$. Also, since $U$ has a dense range it will in fact extend to be unitary.

Use formulas (5.1) and (5.2) to obtain for every polynomial $p$

$$
\begin{aligned}
\left\|p(T) x_{0}\right\|^{2} & =\|p\|_{H^{2}}^{2}+\sum_{k=1}\left\|D p_{k}(T) x_{0}\right\|^{2} \\
& =\|p\|_{H^{2}}^{2}+\sum_{k=1} \frac{1}{2 \pi} \int_{0}^{2 \pi}\left|p_{k}\left(e^{i t}\right)\right|^{2} d \mu(t) \\
& =\|p\|_{H^{2}}^{2}+\sum_{k=1} \frac{1}{2 \pi} \int_{0}^{2 \pi}\left|\left(T_{k} p\right)\left(e^{i t}\right)\right|^{2} d \mu(t) \\
& =\|p\|_{\mu}^{2} .
\end{aligned}
$$

The last identity follows from Theorem 4.1(d).

This concludes the proof of the theorem since it follows from the definition of $U$ that $U M_{z}=T U$.

The following theorem says that $\left(M_{z}, D(\mu)\right)$ is a canonical model for cyclic analytic 2-isometries. 
Theorem 5.2. $\left(M_{z}, D(\mu)\right)$ is unitarily equivalent to $\left(M_{z}, D(\nu)\right)$ if and only if $\mu=\nu$.

Proof. Suppose there is a unitary operator $U: D(\mu) \rightarrow D(\nu)$ such that $U M_{z}=$ $M_{z} U$. We have to show that $\mu=\nu$.

First note that $U 1=1$ because $1 \perp z D(\mu)$ and $1 \perp z D(\nu)$. Hence, for every polynomial $p$ we have $U p=p$. Furthermore $U$ is unitary, thus we have $\|z p\|_{\mu}^{2}-\|p\|_{\mu}^{2}=\|z p\|_{\nu}^{2}-\|p\|_{\nu}^{2}$. By Theorem 4.1 this implies that

$$
\frac{1}{2 \pi} \int_{0}^{2 \pi}\left|p\left(e^{i t}\right)\right|^{2} d \mu(t)=\frac{1}{2 \pi} \int_{0}^{2 \pi}\left|p\left(e^{i t}\right)\right|^{2} d \nu(t),
$$

for all polynomials $p$. Using polarization we obtain

$$
\frac{1}{2 \pi} \int_{0}^{2 \pi} e^{i n t} d \mu(t)=\frac{1}{2 \pi} \int_{0}^{2 \pi} e^{i n t} d \nu(t) \quad \forall n \in \mathbb{Z} .
$$

This clearly implies $\mu=\nu$.

\section{MULTIPLIERS, SIMILARITY, AND QUASISIMILARITY}

The following results about multipliers between $D(\mu)$ spaces are rather technical. However, by a well-known argument due to Shields and Wallen [12], one knows that any operator intertwining $\left(M_{z}, D(\mu)\right)$ and $\left(M_{z}, D(\nu)\right)$ can be represented as multiplication by some analytic function $\varphi$. Using this, the results about multipliers will give us results about quasisimilarity and similarity of $\left(M_{z}, D(\mu)\right)$ and $\left(M_{z}, D(\nu)\right)$.

Lemma 6.1. Let $\mu$ and $\nu$ be two finite positive Borel measures on $\mathbb{T}$. Then

(a) $\left\|z^{n} f\right\|_{\nu}^{2}=\|f\|_{\nu}^{2}+n\|\tilde{f}\|_{P^{2}(\nu)}^{2} \quad \forall f \in D(\nu), n \in \mathbb{N}$.

(b) If $f \in D(\nu), g \in D(\mu)$, and $c>0$ such that $\left\|z^{n} f\right\|_{\nu} \leq c\left\|z^{n} g\right\|_{\mu}$ $\forall n \in \mathbb{N}$, then

$$
\|\tilde{f}\|_{P^{2}(\nu)} \leq c\|\tilde{g}\|_{P^{2}(\mu)} .
$$

Proof. (a) We have $\left\|z^{n} f\right\|_{\nu}^{2}-\|f\|_{\nu}^{2}=\sum_{k=1}^{n}\left(\left\|z^{k} f\right\|_{\nu}^{2}-\left\|z^{k-1} f\right\|_{\nu}^{2}\right)=n\|\tilde{f}\|_{P^{2}(\nu)}^{2}$ by Theorem 4.1(b) and (c).

(b)-From the assumption and part (a) we obtain

$$
\|f\|_{\nu}^{2}+n\|\tilde{f}\|_{P^{2}(\nu)}^{2} \leq c\left\{\|g\|_{\mu}^{2}+n\|\tilde{g}\|_{P^{2}(\mu)}^{2}\right\} \quad \forall n \in \mathbb{N} .
$$

Now divide this inequality by $n$ and let $n \rightarrow \infty$.

The author feels that the equivalence of (b), (c), and (d) of the following theorem are known. However, he has not been able to locate a reference.

Theorem 6.2. Let $\mu, \nu$ be finite positive Borel measures on $\mathbb{T}$. The following are equivalent:

(a) $D(\mu) \subseteq D(\nu)$. 
(b) $\exists c>0$ such that for every polynomial $p$

$$
\frac{1}{2 \pi} \int_{0}^{2 \pi}\left|p\left(e^{i t}\right)\right|^{2} d \nu(t) \leq c \frac{1}{2 \pi} \int_{0}^{2 \pi}\left|p\left(e^{i t}\right)\right|^{2} d \mu(t) .
$$

(c) $\exists c>0$ such that $\varphi_{\nu}(z) \leq c \varphi_{\mu}(z) \quad \forall z \in \mathbb{D}$.

(d) $\nu \ll \mu$ and the Radon-Nikodym derivative $d \nu / d \mu$ is in $L^{\infty}(\mu)$, i.e., $d \nu=h d \mu$ for $h \in L^{\infty}(\mu), h \geq 0$.

Proof. It is clear that (d) implies (c). Also the implication (c) $\Rightarrow$ (a) follows immediately from the definitions. Thus, to finish the proof we have to verify that the implications $(a) \Rightarrow(b),(b) \Rightarrow(c)$, and $(c) \Rightarrow(d)$ are true.

(a) $\Rightarrow(b)$. It follows from the closed graph theorem that the inclusion map from $D(\mu)$ into $D(\nu)$ is continuous, hence there is a constant $c>0$ such that $\|f\|_{\nu} \leq c\|f\|_{\mu}$ for all $f \in D(\mu)$.

Now let $p$ be a polynomial. For $n \in \mathbb{N}$ substitute $f=z^{n} p$ into the previous inequality, and then use Lemma 6.1(b) to deduce that $\|\tilde{p}\|_{P^{2}(\nu)} \leq c\|\tilde{p}\|_{P^{2}(\mu)}$. This implies (6.1).

(b) $\Rightarrow$ (c). Fix $z \in \mathbb{D}$. For every $n \in \mathbb{N}$ define a polynomial $p_{n, z}\left(e^{i t}\right)=$ $\sqrt{1-|z|^{2}} \sum_{k=0}^{n-1} \bar{z}^{k} e^{i k t}$. Then $\left|p_{n, z}\left(e^{i t}\right)\right|^{2}=P\left(z, e^{i t}\right)\left|1-z^{n} e^{-i n t}\right|^{2}$. Substitute this into (6.1) and let $n \rightarrow \infty$ to see that $\varphi_{\nu}(z) \leq c \varphi_{\mu}(z)$

(c) $\Rightarrow$ (d). Define a Borel measure on $\mathbb{T}$ by $\sigma=c \mu-\nu$. Then $d \sigma=$ $c d \mu-d \nu$, hence the assumption (c) implies that $(1 / 2 \pi) \int P\left(z, e^{i t}\right) d \sigma(t)$ is a nonnegative harmonic function. Thus, it follows that $\sigma$ is a positive Borel measure (see e.g. [7, p. 6]). This clearly implies $\nu \ll \mu$, and the RadonNikodým derivative $h=d \nu / d \mu$ satisfies $h\left(e^{i t}\right) \leq c$ a.e. $[\mu]$.

The following corollary immediately follows from Theorem 6.2.

Corollary 6.3. Let $\mu, \nu$ be finite positive Borel measures on $\mathbb{T}$. The following are equivalent:

(a) $D(\mu)=D(\nu)$.

(b) $\exists c, C>0$ such that $c \varphi_{\mu}(z) \leq \varphi_{\nu}(z) \leq C \varphi_{\mu}(z) \quad \forall z \in \mathbb{D}$.

(c) $\nu$ and $\mu$ are mutually absolutely continuous, and the Radon-Nikodym derivative $h=d \nu / d \mu$ satisfies $h, 1 / h \in L^{\infty}(\mu)=L^{\infty}(\nu)$, i.e., $d \nu=$ $h d \mu$ for $h, 1 / h \in L^{\infty}(\mu), h \geq 0$ a.e. $[\mu]$.

Definition 6.4. Given a positive finite Borel measure $\mu$ on $\mathbb{T}$ and a function $f \in D(\mu)$ we define a finite Borel measure $\mu_{f}$ by $d \mu_{f}=|\tilde{f}|^{2} d \mu$.

Note that, if $\mu$ is absolutely continuous, then $\tilde{f}$ coincides with the radial limit function $\hat{f}$ of $f$ (see Proposition 4.3). Thus, if $f \neq 0$, then $|\tilde{f}|^{2}$ is strictly positive, except maybe on a set of Lebesgue measure 0 . This implies that in this case $\mu$ and $\mu_{f}$ are mutually absolutely continuous.

Below we denote the set of multipliers $\{\varphi \in D(\nu): \varphi D(\mu) \subseteq D(\nu)\}$ by $M(D(\mu), D(\nu))$. We shall write $M_{\varphi}$ for the multiplication operator $f \rightarrow \varphi f$. 
Lemma 6.5. Let $\mu, \nu$ be finite positive Borel measures on $\mathbb{T}$, and let $\varphi \in$ $M(D(\mu), D(\nu))$. Then $D(\mu) \subseteq D\left(\nu_{\varphi}\right)$; thus there is $h \in L^{\infty}(\mu), h \geq 0$, a.e. $[\mu]$ such that $d \nu_{\varphi}=h d \mu$.

If $\varphi D(\mu)$ is closed in $D(\nu)$ (equivalently, $M_{\varphi}$ is bounded below), then $D(\mu)=$ $D\left(\nu_{\varphi}\right)$. Thus, in this case $[\mu]=\left[\nu_{\varphi}\right]$ and there is $h \in L^{\infty}(\mu)$ such that $1 / h \in L^{\infty}(\mu)$ and $d \mu=h d \nu_{\varphi}=h|\tilde{\varphi}|^{2} d \nu$.

If $\varphi D(\mu)$ is dense in $D(\nu)$, then $\nu$ and $\nu_{\varphi}$ are mutually absolutely continuous, hence $\mu \ll \nu$.

We shall postpone the proof until after the next corollary, whose first part follows immediately by taking $\mu=\nu$. Note that one always has $M(D(\mu)) \subseteq$ $H^{\infty}$.

Corollary 6.6. If $\varphi \in M(D(\mu))$, then $\tilde{\varphi} \in L^{\infty}(\mu)$. If $M_{\varphi}$ is bounded below, then $1 / \tilde{\varphi} \in L^{\infty}(\mu)$.

Suppose $[\mu]=[m]$ and $\varphi \in M(D(\mu))$ is outer. Then $M_{\varphi}$ is bounded below if and only if $1 / \varphi \in M(D(\mu))$.

Proof. The first part follows from Lemma 6.5.

Suppose that $\mu$ and $m$ are mutually absolutely continuous measures. If $1 / \varphi \in M(D(\mu))$, then $M_{\varphi}$ is invertible and, in particular, it is bounded below. So assume that $M_{\varphi}$ is bounded below. Of course, $\varphi \in D(\mu)$. By the first part and Proposition 4.3, $1 / \varphi \in H^{\infty}$. We have $(1 / \varphi)^{\prime}=-\left(1 / \varphi^{2}\right) \varphi^{\prime}$, hence $1 / \varphi \in$ $D(\mu)$. If $p$ is a polynomial, then $(1 / \varphi) p \in D(\mu)$ and $\|p\|_{\mu}=\left\|M_{\varphi}(1 / \varphi) p\right\|_{\mu} \geq$ $c\|(1 / \varphi) p\|_{\mu}$, i.e., $1 / \varphi \in M(D(\mu))$.

Proof (of Lemma 6.5). The assumption implies that there is a $c>0$ such that $\|p \varphi\|_{\nu} \leq c\|p\|_{\mu}$ for all polynomials $p$. By Lenma 6.1(b) and Theorem 4.1 it follows that

$$
\frac{1}{2 \pi} \int|p|^{2} d \nu_{\varphi}=\frac{1}{2 \pi} \int|p|^{2}|\tilde{\varphi}|^{2} d \nu \leq c \frac{1}{2 \pi} \int|p|^{2} d \mu .
$$

Thus Theorem 6.2 implies the first part of the lemma.

If $M_{\varphi}$ is bounded below, then there are constants $c, C>0$ such that $c\|p\|_{\mu} \leq\|p \varphi\|_{\nu} \leq C\|p\|_{\mu}$ for all polynomials $p$. The same argument as in the first part of the proof implies that $\mu$ and $\nu_{\varphi}$ are mutually absolutely continuous and there is an $h \in L^{\infty}\left(\nu_{\varphi}\right)$ such that $1 / h \in L^{\infty}\left(\nu_{\varphi}\right)$ and $d \mu=h d \nu_{\varphi}=$ $h|\tilde{\varphi}|^{2} d \nu$. By Corollary 6.3 this means $D(\mu)=D\left(\nu_{\varphi}\right)$.

To finish the proof we have to show that $\nu_{\varphi}$ and $\nu$ are mutually absolutely continuous if $\varphi D(\mu)$ is dense in $D(\nu)$. We note that it follows from the assumption that $\varphi$ must be a cyclic vector for $\left(M_{z}, D(\nu)\right)$. Thus the verification of the following claim will conclude the proof of the lemma.

Claim. If $\varphi$ is cyclic in $D(\nu)$, then $\nu\left(\left\{e^{i t}: \tilde{\varphi}\left(e^{i t}\right)=0\right\}\right)=0$. Thus, $\nu_{\varphi}$ and $\nu$ are mutually absolutely continuous. 
Proof (of the claim). We may assume that $\nu \neq 0$. Let $E=\left\{e^{i t}: \tilde{\varphi}\left(e^{i t}\right)=0\right\}$ and assume that $\nu(E)>0$. We have to show that $\varphi$ is not cyclic in $D(\nu)$.

If $p$ is a polynomial, then

$$
\begin{aligned}
0 & <\frac{1}{2 \pi} \nu(E)=\frac{1}{2 \pi} \int_{E}|p \tilde{\varphi}-1|^{2} d \nu \\
& \leq \frac{1}{2 \pi} \int_{\mathbb{T}}|p \tilde{\varphi}-1|^{2} d \nu \leq C\|p \varphi-1\|_{\nu}^{2} .
\end{aligned}
$$

Thus, it follows that $\varphi$ cannot be cyclic in $D(\nu)$.

Recall that two operators $T_{1} \in \mathscr{L}\left(\mathscr{H}_{1}\right)$ and $T_{2} \in \mathscr{L}\left(\mathscr{H}_{2}\right)$ are said to be similar if there is an invertible operator $S \in \mathscr{L}\left(\mathscr{H}_{1}, \mathscr{H}_{2}\right)$ such that $S T_{1}=T_{2} S$. $T_{1}$ and $T_{2}$ are said to be quasisimilar if there are injective operators $X \in$ $\mathscr{L}\left(\mathscr{H}_{1}, \mathscr{H}_{2}\right)$ and $Y \in \mathscr{L}\left(\mathscr{H}_{2}, \mathscr{H}_{1}\right)$ such that both $X$ and $Y$ have a dense range and the intertwining relations $X T_{1}=T_{2} X$ and $T_{1} Y=Y T_{2}$ hold.

Note that, if $T_{1}$ is analytic and quasisimilar to $T_{2}$, then $T_{2}$ is analytic as well. Indeed, suppose that $\bigcap_{n>0} T_{1}^{n} \mathscr{H}_{1}=\{0\}$ and $Y$ is $1-1$ such that $T_{1} Y=$ $Y T_{2}$. Let $\mathscr{M}=\bigcap_{n>0} T_{2}^{n} \mathscr{H}_{2}$. We have $Y \mathscr{M} \subseteq \bigcap_{n>0} Y T_{2}^{n} \mathscr{H}_{2}=\bigcap_{n>0} T_{1}^{n} Y \mathscr{H}_{2} \subseteq$ $\bigcap_{n>0} T_{1}^{n} \mathscr{H}_{1}=\{0\} . Y$ is $1-1$, hence it follows that $\mathscr{M}=\{0\}$. Also, if $T_{1}$ has a cyclic vector, then $T_{2}$ must have a cyclic vector as well.

Thus, if $\left(M_{z}, D(\mu)\right)$ is quasisimilar to a 2-isometry $T$, then $T$ must be a cyclic analytic 2-isometry. By Theorem 5.1, $T$ is unitarily equivalent to $\left(M_{z}, D(\nu)\right)$ for some $\nu$. The following theorem gives a necessary condition on $\mu$ and $\nu$ for $\left(M_{z}, D(\mu)\right)$ and $\left(M_{z}, D(\nu)\right)$ to be quasisimilar. We shall see later (see the remark after Proposition 6.8) that this condition is not sufficient.

Theorem 6.7. If $\left(M_{z}, D(\mu)\right)$ and $\left(M_{z}, D(\nu)\right)$ are quasisimilar, then $\mu$ and $\nu$ are mutually absolutely continuous.

Proof. Let $X \in \mathscr{L}(D(\mu), D(\nu))$ and $Y \in \mathscr{L}(D(\nu), D(\mu))$ be the operators that institute the quasisimilarity between $\left(M_{z}, D(\mu)\right)$ and $\left(M_{z}, D(\nu)\right)$. Then $X$ and $Y$ intertwine $\left(M_{z}, D(\mu)\right)$ and $\left(M_{z}, D(\nu)\right)$, hence they can be represented as multiplications by some analytic functions $\varphi_{1}, \varphi_{2}$. The polynomials are dense in $D(\mu)$ and $D(\nu)$; thus we have $\varphi_{1} D(\mu) \subseteq D(\nu)$ and $\varphi_{2} D(\nu) \subseteq D(\mu)$, where the containments are dense. It now follows from Lemma 6.5 that $\mu$ and $\nu$ must be mutually absolutely continuous.

In the special case where one of the measures is Lebesgue measure we can prove the following.

Proposition 6.8. Suppose $f \in D \cap H^{\infty}$ is an outer function. If $\left(M_{z}, D\left(m_{f}\right)\right)$ is quasisimilar to $\left(M_{z}, D\right)$, then $f$ is cyclic in $D$.

Proof. $f \in H^{\infty}$ implies that $|\tilde{f}|^{2} \in L^{\infty}(m)$; thus by Theorem 6.2 we have $D \subseteq$ $D\left(m_{f}\right)$. As in the proof of the previous theorem it follows from the hypothesis that there is a function $g \in D$ such that $g D\left(m_{f}\right) \subseteq D$ densely. $g$ must be 
cyclic in $D$, and in particular, it must be an outer function. Furthermore, we have $g D \subseteq g D\left(m_{f}\right) \subseteq D$, i.e., $g$ is a multiplier of the Dirichlet space.

Now let $h=g / f$. Then $h$ is an outer function and $|\tilde{g}|^{2} d t=|\hat{g}|^{2} d t=$ $|\hat{h}|^{2}|\hat{f}|^{2} d t=|\hat{h}|^{2}|\tilde{f}|^{2} d t$. The containment $g D\left(m_{f}\right) \subseteq D$ implies by Lemma 6.5 that $|\hat{h}|^{2} \in L^{\infty}(m)$, i.e., $h \in H^{\infty}$. Hence we have $|g(z)| \leq\|h\|_{\infty}|f(z)|$ for every $z \in \mathbb{D}$. The fact that $g$ is a multiplier and cyclic together with Corollary 1 of [3] now implies that $f$ must be cyclic as well.

We see that, if $f \in D \cap H^{\infty}$ is an outer function which is not cyclic in $D$, then $\left(M_{z}, D\left(m_{f}\right)\right)$ and $\left(M_{z}, D\right)$ are not quasisimilar even though $m$ and $m_{f}$ are mutually absolutely continuous. The existence of noncyclic outer functions in $D$ is a well-known fact. That there are bounded noncyclic outer functions in $D$ follows from the results in [10].

In Theorem 7.5 we shall see that, if $f$ is a rational function with poles off the closed unit disc, then $\left(M_{z}, D\left(m_{f}\right)\right)$ and $\left(M_{z}, D\right)$ are quasisimilar.

If $\left(M_{z}, D(\mu)\right)$ and $\left(M_{z}, D(\nu)\right)$ are similar, then the similarity must be given as multiplication by some analytic function $\varphi$ and $\varphi D(\mu)=D(\nu)$. Thus the following corollary follows immediately from Lemma 6.5. We state it again for clarity.

Corollary 6.9. If $\left(M_{z}, D(\mu)\right)$ and $\left(M_{z}, D(\nu)\right)$ are similar and $\varphi \in D(\nu)$ such that $\varphi D(\mu)=D(\nu)$, then $\mu$ and $\nu$ are mutually absolutely continuous and there is $h \in L^{\infty}(\mu), h \geq 0$, a.e. [ $\mu$ ] such that $1 / h \in L^{\infty}(\mu)$ and $d \mu=h|\tilde{\varphi}|^{2} d \nu$, i.e., $\varphi D\left(\nu_{\varphi}\right)=D(\nu)$.

We shall conclude this section with a theorem that indicates how special the Dirichlet shift is among the 2 -isometries. Note that it follows from Corollary 6.7 that $\left(M_{z}, D(\mu)\right)$ is quasisimilar to the unilateral shift $\left(=\left(M_{z}, H^{2}\right)\right)$ if and only if $\mu=0$.

Theorem 6.10. If $\mu \neq 0$ and $\left(M_{z}, D(\mu)\right)$ is similar to a weighted shift operator, then it is similar to the Dirichlet shift.

Proof. Let $\left\{e_{n}\right\}$ be an orthonormal basis for the Hilbert space $\mathscr{H}$ and $\left\{w_{n}\right\}$ be a sequence of nonnegative numbers such that $T e_{n}=w_{n} e_{n+1}$ defines a bounded weighted shift operator on $\mathscr{H}$. Assume that $T$ is similar to $\left(M_{z}, D(\mu)\right)$ via the similarity $S \in \mathscr{L}(\mathscr{H}, D(\mu))$, i.e., $M_{z} S=S T$. Clearly $\left(M_{z}, D(\mu)\right)$ is injective; thus we may assume that none of the weights are zero.

Below $B \in \mathscr{L}\left(D(\mu), P^{2}(\mu)\right)$ will denote the operator from Theorem 4.1. Recall that $\operatorname{ker} B \in \operatorname{Lat}\left(M_{z}, D(\mu)\right)$ and $\operatorname{ker} B \neq D(\mu)$ since $\mu \neq 0 . e_{0}$ is a cyclic vector for $T$, hence $S e_{0}$ must be cyclic for $\left(M_{z}, D(\mu)\right)$. It follows that $S e_{0} \notin \operatorname{ker} B$.

We shall prove that $T$ is similar to the Dirichlet shift by using Theorem 2 (b) of [11, p. 54]. It says that two weighted shift operators with positive weight sequences $\left\{w_{n}\right\}$ and $\left\{v_{n}\right\}$ are similar if and only if the sequence 
$\left\{w_{0} w_{1} \cdots w_{n} / v_{0} v_{1} \cdots v_{n}\right\}_{n \geq 0}$ is bounded away from zero and infinity independently of $n$.

The weights of the Dirichlet shift are $v_{j}=\sqrt{(j+2) /(j+1)}, j \geq 0$. Hence $\prod_{j=0}^{n-1} v_{j}^{2}=n+1$ for every $n \in \mathbb{N}$. Thus,

$$
\begin{aligned}
0 & <C_{1} \leq \frac{\left\|S e_{0}\right\|_{\mu}^{2}+n\left\|B S e_{0}\right\|_{P^{2}(\mu)}^{2}}{(n+1)\|S\|^{2}} \\
& =\frac{\left\|M_{z}^{n} S e_{0}\right\|_{\mu}^{2}}{(n+1)\|S\|^{2}} \quad(\text { Lemma 6.1) } \\
& =\frac{\left\|S T^{n} e_{0}\right\|^{2}}{(n+1)\|S\|^{2}} \leq \frac{\left\|T^{n} e_{0}\right\|^{2}}{n+1}=\frac{\prod_{j=0}^{n-1} w_{j}^{2}}{\prod_{j=0}^{n-1} v_{j}^{2}} \\
& \leq \frac{\left\|S^{-1}\right\|^{2}\left\|S T^{n} e_{0}\right\|^{2}}{n+1}=\frac{\left\|S^{-1}\right\|^{2}\left\|M_{z}^{n} S e_{0}\right\|_{\mu}^{2}}{n+1} \\
& =\frac{\left\|S^{-1}\right\|^{2}\left(\left\|S e_{0}\right\|_{\mu}^{2}+n\left\|B S e_{0}\right\|_{P^{2}(\mu)}^{2}\right)}{n+1} \leq C_{2}<\infty .
\end{aligned}
$$

Hence it follows from the above quoted theorem that $T$ is similar to the Dirichlet shift.

\section{INVARIANT SUBSPACES}

If $f \in D(\mu)$ and $f D(\nu)$ is contained and closed in $D(\mu)$, then it is an invariant subspace of $\left(M_{z}, D(\mu)\right)$. By Lemma 6.5 we can substitute $\mu_{f}$ for $\nu$, i.e., in this case $\mathscr{M}=f D\left(\mu_{f}\right)$ is an invariant subspace of $\left(M_{z}, D(\mu)\right)$. Of course we have $\operatorname{dim} \mathscr{M} \ominus z \mathscr{M}=1$ (see Theorem 5.1).

Conversely, if $\mathscr{M}$ is an invariant subspace of $\left(M_{z}, D(\mu)\right)$ which satisfies $\operatorname{dim} \mathscr{M} \ominus z \mathscr{M}=1$, then $M_{z} \mid \mathscr{M}$ is a 2-isometry that satisfies the hypothesis of Theorem 5.1. Thus, there is a positive finite Borel measure $\nu$ on $\mathbb{T}$ such that $\left(M_{z}, D(\nu)\right)$ is unitarily equivalent to $M_{z} \mid \mathscr{M}$. The following theorem will explain the relationship between $\mu$ and $\nu$.

Theorem 7.1. Let $\mathscr{M}$ be an invariant subspace of $\left(M_{z}, D(\mu)\right)$ that satisfies $\operatorname{dim} \mathscr{M} \ominus z \mathscr{M}=1$.

If $f \in \mathscr{M} \ominus z \mathscr{M},\|f\|_{\mu}=1$, then $\mathscr{M}=f D\left(\mu_{f}\right)$ and $\|f g\|_{\mu}=\|g\|_{\mu_{f}}$ for every $g \in D\left(\mu_{f}\right)$.

In particular, every invariant subspace of the Dirichlet shift $\left(M_{z}, D\right)$ is of the form $f D\left(m_{f}\right)$.

Proof. The polynomials are dense in $D\left(\mu_{f}\right)$. Furthermore, by Theorem 1 of [9] we know that the polynomial multiples of $f$ are dense in $\mathscr{M}$. Thus, it is enough to show that $\|f p\|_{\mu}=\|p\|_{\mu}$ for every polynomial $p$.

Recall that for a polynomial $p(z)=\sum_{n=0} \hat{p}(n) z^{n}$ and for a nonnegative integer $k$ we use the notation $p_{k}=T_{k} p$ to denote the truncated polynomial 
$p_{k}(z)=\sum_{n=k} \hat{p}(n) z^{n}$. One has $\mathscr{M} \ominus z \mathscr{M}=\operatorname{ker}\left(M_{z} \mid \mathscr{M}^{*}\right.$. Thus, Lemma 2.1 applied with the operator $T=M_{z} \mid \mathscr{M}$ and the vector $x_{0}=f$ yields

$$
\begin{aligned}
\|p f\|_{\mu}^{2} & =\|p\|_{H^{2}}^{2}+\sum_{k=1}\left(\left\|z p_{k} f\right\|_{\mu}^{2}-\left\|p_{k} f\right\|_{\mu}^{2}\right) \\
& =\|p\|_{H^{2}}^{2}+\sum_{k=1} \frac{1}{2 \pi} \int_{0}^{2 \pi}\left|p_{k}\left(e^{i t}\right)\right|^{2}\left|\tilde{f}\left(e^{i t}\right)\right|^{2} d \mu(t) \\
& =\|p\|_{\mu_{f}}^{2} .
\end{aligned}
$$

Here the last two equalities follow from Theorem 4.1.

The statement about the invariant subspaces of the Dirichlet shift now follows immediately because by Theorem 2 of [10] $\operatorname{dim} \mathscr{M} \ominus z \mathscr{M}=1$ for every nonzero invariant subspace of $\left(M_{z}, D\right)$.

Thus, in order to precisely describe all invariant subspaces of the Dirichlet shift one needs to identify all functions $f \in D$ which satisfy $f \perp z^{n} f$ for all $n \in \mathbb{N}$. This may be hard and it may be easier to first determine all $f \in D$ such that $f D\left(m_{f}\right)$ is closed in $D$. We now give an example.

Example 7.2. Let $r>0, g(z)=e^{-r(1+z) /(1-z)}$, and $f(z)=(1-z) g(z)$.

Since $m$ is Lebesgue measure and $g$ is an inner function, it follows from Proposition 4.3 that $\left|\tilde{f}\left(e^{i t}\right)\right|=\left|1-e^{i t}\right|$. Thus, $d m_{f}(t)=\left|1-e^{i t}\right|^{2} d t$. This is the same measure as in Example 3.2 and we recall that $\varphi_{m_{f}}(z)=2(1-\operatorname{Re} z)=$ $|1-z|^{2}+1-|z|^{2}$.

Claim. $f D\left(m_{f}\right)$ is an invariant subspace of $\left(M_{z}, D\right)$.

We know that the polynomials are dense in $D\left(m_{f}\right)$; hence it will suffice to show that there are constants $c, C>0$ such that for every polynomial $p$

$$
\begin{aligned}
& c\left(\|(1-z) g p\|_{H^{2}}^{2}+\iint_{\mathbb{D}}\left|((1-z) g p)^{\prime}(z)\right|^{2} d A(z)\right) \\
& \leq\|p\|_{H^{2}}^{2}+\iint_{\mathbb{D}}\left|p^{\prime}(z)\right|^{2} \varphi_{m_{f}}(z) d A(z) \\
& \leq C\left(\|(1-z) g p\|_{H^{2}}^{2}+\iint_{\mathbb{D}}\left|((1-z) g p)^{\prime}(z)\right|^{2} d A(z)\right) .
\end{aligned}
$$

To verify this it will be convenient to use Carleson's representation formula for the Dirichlet integral (see [4]). We apply that formula to $(1-z) g p$ and to $(1-z) p$ and, since $g(z)=e^{-r(1+z) /(1-z)}$ we obtain

$$
\iint_{\mathbb{D}}\left|((1-z) g p)^{\prime}(z)\right|^{2} d A(z)=\frac{r}{2}\|p\|_{H^{2}}^{2}+\iint_{\mathbb{D}}\left|((1-z) p)^{\prime}(z)\right|^{2} d A(z) .
$$


Thus,

$$
\begin{aligned}
\|(1- & z) g p \|_{H^{2}}^{2}+\iint_{\mathbb{D}}\left|((1-z) g p)^{\prime}(z)\right|^{2} d A(z) \\
& \leq\left(4+\frac{r}{2}\right)\|p\|_{H^{2}}^{2}+\iint_{\mathbb{D}}\left|((1-z) p)^{\prime}(z)\right|^{2} d A(z) \\
& \leq c\left(\|p\|_{H^{2}}^{2}+\iint_{\mathbb{D}}\left|p^{\prime}(z)\right|^{2}|1-z|^{2} d A(z)+\iint_{\mathbb{D}}|p(z)|^{2} d A(z)\right) \\
& \leq c\left(\|p\|_{H^{2}}^{2}+\iint_{\mathbb{D}}\left|p^{\prime}(z)\right|^{2} \varphi_{m_{f}}(z) d A(z)\right)
\end{aligned}
$$

since $|1-z|^{2} \leq \varphi_{m_{f}}(z)$ and because the $L_{a}^{2}$-norm is smaller than the $H^{2}$-norm. This establishes the first half of (7.1).

Now compare (7.1) and (7.2) and note that in order to finish the proof it will be sufficient to show that

$$
\iint_{\mathbb{D}}\left|p^{\prime}(z)\right|^{2} \varphi_{m_{f}}(z) d A(z) \leq C\left(\iint_{\mathbb{D}}\left|((1-z) p)^{\prime}(z)\right|^{2} d A(z)+\|p\|_{H^{2}}^{2}\right) .
$$

We have $(1-z) p^{\prime}=((1-z) p)^{\prime}+p$; thus

$$
\begin{aligned}
\iint_{\mathbb{D}}\left|p^{\prime}(z)\right|^{2} \varphi_{m_{f}}(z) d A(z)=\iint_{\mathbb{D}}\left|p^{\prime}(z)\right|^{2}\left(|1-z|^{2}+\left(1-|z|^{2}\right)\right) d A(z) \\
\leq c\left(\iint_{\mathbb{D}}\left|p^{\prime}(z)(1-z)\right|^{2} d A(z)+\|p\|_{H^{2}}^{2}\right) \quad(\text { see } \S 2) \\
\quad \leq c\left(\iint_{\mathbb{D}}\left|((1-z) p)^{\prime}(z)\right|^{2} d A(z)+\iint_{\mathbb{D}}|p(z)|^{2} d A(z)+\|p\|_{H^{2}}^{2}\right) \\
\quad \leq c\left(\iint_{\mathbb{D}}\left|((1-z) p)^{\prime}(z)\right|^{2} d A(z)+\|p\|_{H^{2}}^{2}\right) \cdot
\end{aligned}
$$

In $\S 6$ we have obtained some necessary conditions for $\left(M_{z}, D(\mu)\right)$ and $\left(M_{z}, D(\nu)\right)$ to be similar operators and we have seen that the only weighted shifts that they can be similar to are the unilateral shift or the Dirichlet shift. For restrictions of the Dirichlet shift to an invariant subspace we can now easily prove the following proposition, which might be considered somewhat surprising.

Proposition 7.3. Let $D=D(m)$ denote the Dirichlet space. If $\mathscr{M} \in \operatorname{Lat}\left(M_{z}, D\right)$, then $M_{z} \mid \mathscr{M}$ is similar to a weighted shift operator if and only if $\mathscr{M}$ is generated by a finite Blaschke product. In this case it is similar to the Dirichlet shift.

Proof. If $f \in D$, then let $[f]$ denote the smallest invariant subspace of $\left(M_{z}, D\right)$ containing $f$.

Multiplication by a finite Blaschke product $B$ is bounded below in $\mathscr{L}(D)$, hence it is clear that $[B]=B D$ and that $M_{z} \mid[B]$ is similar to the Dirichlet shift.

To prove the converse assume that $\mathscr{M} \in \operatorname{Lat}\left(M_{z}, D\right), \mathscr{M} \neq(0)$. Let $f \in$ $\mathscr{M} \ominus z \mathscr{M},\|f\|_{m}=1$. Then $M_{z} \mid \mathscr{M}$ is unitarily equivalent to $\left(M_{z}, D\left(m_{f}\right)\right)$. 
$m_{f} \neq 0$, thus by Theorem $6.10, M_{z} \mid \mathscr{M}$ is similar to $\left(M_{z}, D\right)$. Since $\mathscr{M} \subseteq D$ the similarity must be given by a multiplier $\varphi \in M(D)$, which is bounded below. Let $\varphi=S \varphi_{1}$ be the inner-outer factorization of $\varphi$. Multiplication by an inner factor always increases the Dirichlet norm (see [4]); thus $\varphi_{1} \in M(D)$. Furthermore, by Proposition 4.3, $\tilde{\varphi}=\hat{\varphi}=\hat{\varphi}_{1}=\tilde{\varphi}_{1}$ in $L^{2}(m)$. It follows from Corollary 6.6 that $1 / \tilde{\varphi}_{1} \in L^{\infty}(m)$ and hence $1 / \varphi_{1} \in H^{\infty}$. This implies $1 / \varphi_{1} \in D$ and $\|S\|_{m}=\left\|M_{\varphi}\left(1 / \varphi_{1}\right)\right\|_{m} \leq c\left\|1 / \varphi_{1}\right\|_{m}$. Thus $S \in D$, i.e., $S=B$ must be a finite Blaschke product. This implies that $M_{B}$ is a multiplier and bounded below. Hence $M_{\varphi_{1}}$ is bounded below. By Corollary 6.6, $1 / \varphi_{1} \in M(D)$ and $\mathscr{M}=[B]$.

We shall now conclude this section by showing that quasisimilarity of restrictions of $\left(M_{z}, D\right)$ to invariant subspaces is more common than similarity. Recall that the disc algebra is the space of all analytic functions on $\mathbb{D}$ that extend to be continuous on the closure of $\mathbb{D}$.

Lemma 7.4. Suppose $f$ is analytic on $\mathbb{D}$ and there is an $\varepsilon>0$ and $a c>0$ such that for all $z \in \mathbb{D}$

$$
\left|f^{\prime}(z)\right|^{2}\left(1-|z|^{2}\right)^{1-\varepsilon} \leq c .
$$

Then $f$ is in the disc algebra and $f D\left(m_{f}\right) \subseteq D$ and $D \subseteq D\left(m_{f}\right)$.

Proof. It follows from a theorem of Hardy and Littlewood (see e.g. Duren, Theory of $H^{p}$ spaces, p. 74) and the assumption that $f$ is in the disc algebra (and satisfies a Lipschitz condition). This implies that $|\tilde{f}|^{2} \in L^{\infty}(m)$. Thus by the definition of $m_{f}$ and by Theorem 6.2 we obtain $D \subseteq D\left(m_{f}\right)$.

We shall finish the proof by showing that

$$
\iint_{\mathbb{D}}\left|(p f)^{\prime}(z)\right|^{2} d A(z) \leq c\|p\|_{m_{f}}^{2} .
$$

For any analytic function $f$ on $\mathbb{D}$ one has $|f(z)|^{2} \leq \varphi_{m_{f}}(z)$ for every $z \in \mathbb{D}$. Thus,

$$
\begin{aligned}
\iint_{\mathbb{D}}\left|(p f)^{\prime}(z)\right|^{2} d A(z) \\
\quad \leq C\left(\iint_{\mathbb{D}}\left|p^{\prime}(z) f(z)\right|^{2} d A(z)+\iint_{\mathbb{D}}\left|p(z) f^{\prime}(z)\right|^{2} d A(z)\right) \\
\quad \leq C\left(\iint_{\mathbb{D}}\left|p^{\prime}(z)\right|^{2} \varphi_{m_{f}}(z) d A(z)+c \iint_{\mathbb{D}}|p(z)|^{2}\left(1-|z|^{2}\right)^{-1+\varepsilon} d A(z)\right) \\
\quad \leq C\left(\iint_{\mathbb{D}}\left|p^{\prime}(z)\right|^{2} \varphi_{m_{f}}(z) d A(z)+c\|p\|_{H^{2}}^{2}\right) \leq C\|p\|_{m_{f}}^{2} \cdot \quad \square
\end{aligned}
$$

Theorem 7.5. If $f$ is a rational function with poles off the closed unit disc, then $\left(M_{z}, D\left(m_{f}\right)\right)$ is quasisimilar to $\left(M_{z}, D\right)$.

Proof. First note that one can find a finite Blaschke product $B$ and a rational function $g$ with no poles in $\mathbb{D}^{-}$and no zeros in $\mathbb{D}$ such that $f=B g$. By 
Corollary 6.3, $\left(M_{z}, D_{m_{f}}\right)$ is similar to $\left(M_{z}, D_{m_{g}}\right)$, so in order to finish the proof of the theorem it suffices to show that $\left(M_{z}, D_{m_{g}}\right)$ is quasisimilar to $\left(M_{z}, D\right)$.

But $g$ satisfies the hypothesis of Lemma 7.4 and clearly $D$ is dense in $D\left(m_{g}\right)$ (it contains the polynomials), hence we have to show that $g D\left(m_{g}\right)$ is dense in $D$. This will be the case if $g$ is a cyclic vector in $D$.

To see that $g$ is cyclic one needs to recall results of Leon Brown and Allen Shields. In fact, the conclusion is immediate from [Proposition 8, p. 275, Lemma 8, and Proposition 13, p. 289].

We note Example 7.2, Theorem 7.3, and Theorem 7.5 imply that there are quasisimilar analytic 2-isometries that are not similar to one another. Indeed, if $p(z)=1-z$, then by Theorem 7.5, $\left(M_{z}, D\left(m_{p}\right)\right)$ is quasisimilar to the Dirichlet shift. On the other hand, by Example 7.2 we know that $\left(M_{z}, D\left(m_{p}\right)\right)$ is similar to $M_{z} \mid \mathscr{M}$, where $\mathscr{M} \in \operatorname{Lat}\left(M_{z}, D\right)$ and all functions in $\mathscr{M}$ have a singular inner factor. Now, if $M_{z} \mid \mathscr{M}^{2}$ were similar to $\left(M_{z}, D\right)$, then by Theorem 7.3, $\mathscr{M}$ would have to be generated by a finite Blaschke product. This is clearly impossible. Thus $\left(M_{z}, D\left(m_{p}\right)\right)$ is not similar to the Dirichlet shift.

This observation is in contrast to the fact that quasisimilar isometries must be unitarily equivalent.

\section{REFERENCES}

1. J. Agler, A disconjugacy theorem for Toeplitz operators, Amer. J. Math. 112 (1990), 1-14.

2. J. Bram, Subnormal operators, Duke Math. J. 22 (1955), 75-94.

3. L. Brown and A. L. Shields, Cyclic vectors in the Dirichlet space, Trans. Amer. Math. Soc. 285 (1984), 269-304.

4. L. Carleson, A representation formula for the Dirichlet integral, Math. Z. 73 (1960), 190196.

5. M. J. Cowen and R. G. Douglas, Complex geometry and operator theory, Acta Math. 141 (1978), 187-261.

6. P. R. Halmos, Ten problems in Hilbert space, Bull. Amer. Math. Soc. 76 (1970), 887-933.

7. P. Koosis, Introduction to $H^{p}$-spaces, Cambridge Univ. Press, 1980.

8. S. Richter, Invariant subspaces in Banach spaces of analytic functions, Trans. Amer. Math. Soc. 304 (1987), 585-616.

9. __ Invariant subspaces of the Dirichlet shift, J. Reine Angew. Math. 386 (1988), 205-220.

10. S. Richter and A. L. Shields, Bounded analytic functions in the Dirichlet space, Math. Z. 198 (1988), 151-159.

11 A. L. Shields, Weighted shift operators and analytic function theory, Math. Surveys, vol. 13, Amer. Math. Soc., Providence, RI, 1974.

12. A. L. Shields and L. J. Wallen, The commutants of certain Hilbert space operators, Indiana Univ. Math. J. 20 (1971), 777-788.

13. G. D. Taylor, Multipliers on $D_{\alpha}$, Trans. Amer. Math. Soc. 123 (1966), 229-240.

Department of Mathematics, University of Tennessee, Knoxville, Tennessee 379961300 